This is a postprint/Accepted version of the following published document:

Cuadra, A.; Huete, C.; Vera, M. Effect of equivalence ratio fluctuations on planar detonation discontinuities. In: Journal of fluid mechanics, 903, A30, Nov. 2020, 39 pp.

DOI: https://doi.org/10.1017/ifm.2020.651

(C) The Author(s), 2020. 


\title{
Effect of equivalence ratio fluctuations on planar detonation discontinuities
}

\author{
Alberto Cuadra ${ }^{1}$, César Huete ${ }^{1} \dagger$, Marcos Vera ${ }^{1}$ \\ ${ }^{1}$ Grupo de Mecánica de Fluidos, Universidad Carlos III, Av. Universidad 30, 28911, Leganés, \\ Spain
}

(Received xx; revised xx; accepted $\mathrm{xx}$ )

We propose a linear asymptotic theory to describe the propagation of planar detonation fronts through heterogeneous mixtures of reactive gases consisting of random fluctuations in the fuel mass fraction. The analysis starts with the derivation of the transfer functions that relate the upstream fuel-mass-fraction inhomogeneities with the burntgas perturbations via normal mode analysis. These results are then used in a Fourier analysis of a detonation wave interacting with two- and three-dimensional isotropic heterogeneous fields. This yields integral formulae for the turbulent kinetic energy, sonic energy and averaged vorticity and entropy production rates. Second-order corrections for the turbulent Rankine-Hugoniot conditions are also obtained, along with analytical expressions for the deviation of the detonation velocity with respect to that of the equivalent homogeneous mixture. Upstream inhomogeneities are found to speed up the detonation front in the vast majority of scenarios studied, with a velocity amplification factor that depends on the properties of the fuel-air mixture, particularly on the variation of the density and the heat release with the fuel mass fraction.

\section{Introduction}

Detonation waves have been explored extensively for propulsion applications given their theoretical advantage over deflagrative pre-mixed combustion. For instance, rotating detonation engines, which do not operate under the Brayton Cycle, benefit from the additional work extraction from the cycle and a decrease in the physical size of the combustor (Huff et al. 2019). Premixing of fuel and air may be necessary due to finite ignition delay times compared to the residence time of the fluid particles in the combustion chamber. Consequently, it is of paramount importance to understand how standing detonation waves may be affected by the inhomogeneities of the reactive gas mixture to design efficient supersonic combustors (Pratt et al. 1991; Urzay 2018; Frolov et al. 2019). However, many challenges must be surmounted before detonation-based engines could be presented as a competitive alternative. Some hurdles include the incomplete fuel-air mixing at the molecular level, the stability of the detonation, and the strong noise generated by the system even in highly idealized configurations (Kailasanath 2000, 2003). Non-idealized configurations representative of more realistic systems may include additional complexities, such as detonation-wall interaction, confinement, turbulence, multi-phase flow and non-perfect mixing, in addition to the complexities that arise in the modelling of chemical kinetics and transport properties (Meng et al. 2018).

When the burning process in the premixed environment occurs at high Mach numbers, acoustics play a dominant role in the reactive flow dynamics, as is the case of detonations. Unlike deflagrations, the thickness of the hydrodynamic region affecting the detonation

$\dagger$ Email address for correspondence: chuete@ing.uc3m.es 
front only compares to the detonation thickness because there is no possibility for a preheat layer. Moreover, diffusive effects become subdominant given that the residence time of the reactive mixture in the induction-burning layer is considerably reduced compare with the characteristic diffusion time. Radicals formation and recombination affect the detonation thickness and dynamics, but they do not influence the propagation speed, at least at the first order. When the planar detonation exhibits an unstable propagation pattern (Short \& Quirk 1997; Kasimov \& Stewart 2004; Radulescu et al. 2007; Shepherd 2009 Clavin \& Williams 2012; Kabanov \& Kasimov 2018; Han et al. 2019 Huete \& Vera 2019), the disturbances generated downstream affect the averaged Rankine-Hugoniot (RH) relationships, since the energy employed in the generation of vorticity, entropy and sonic perturbations comes from the only source available in selfsustained detonations: the heat release by combustion. It has been found that upstream heterogeneities play a pivotal role in the detonation dynamics (Williams 1961, Veyssiere \& Khasainov 1995, Kessler et al. 2012, Ettner et al. 2013; Prakash et al. 2019b a |Prakash \& Raman 2019). Enhancement of the propagation speed has been observed in gaseous mixtures as a result of the turbulence induced by the detonation front wrinkling (Mi et al. $2017 a$ b Prakash \& Raman 2019, Meadows \& Subramanian 2019). When moving through fuel-air sprays with large droplets, detonations exhibit a deficit in the propagation velocity compared to the gas-phase velocity (Kailasanath 2006). Similar effects have been observed in detonations moving through gaseous reactive media with water clouds (Watanabe et al. 2019, 2020). However, a clear correlation between the upstream properties of the heterogeneous mixture and the detonation propagation velocity has not been presented to date. This work aims to shed further light, from a theoretical framework, on the hydrodynamical aspects that contribute to the modification of detonation velocity when the detonation wave propagates through a non-uniform mixture of reactive gases. The linear-interaction analysis predicts a second-order propagation velocity augmentation for weakly overdriven detonations independently of the type of perturbations generated by the equivalence ratio fluctuations, i.e., positive or negative perturbations in the upstream density and/or heat of combustion fields. For strongly overdriven detonations, a weak deficit in the propagation speed is found only when upstream perturbations in the equivalence ratio are dominated by heat release perturbations.

Earlier work on the interaction of small-amplitude perturbations with detonation waves can be found in Massa et al. (2011); Massa \& Lu (2011); Jin et al. (2016); Hussein (2018); Jackson et al. (1990, 1993); Huete et al. (2013, 2014); Griffond (2005); Huete et al. (2017). When the problem is addressed numerically, chemical kinetic models can be implemented (Massa et al. 2011; Massa \& Lu 2011; Jin et al. 2016, Hussein 2018), which allows the description of the detonation unstable modes. Numerical studies by Massa et al. (2011); Massa \& Lu (2011) demonstrate enhanced interactions between turbulence and detonations when sizes of the inhomogeneities and the unperturbed reaction zones are comparable. For certain values of the activation energy, small scales of the postshock perturbation modes increase unbounded owing to acoustic resonance. In different conditions, however, turbulence may cushion the detonation self-induced oscillations by forcing the reaction wave to adapt to the ever-changing upstream conditions. Under this stable condition, the present theory and that employed in Jackson et al. (1990, 1993); Huete et al. (2013, 2014, 2017) may be applied. Employing the fast-reaction limit allows the detonation wave to be treated as a single discontinuity front, and the chemical process exclusively enters through the non-adiabatic RH curve. The motivation was to improve descriptions of the influences of compressible turbulence on detonation propagation and, in particular, to determine how passage of a planar detonation modifies the turbulence. This work complements these fundamental studies, particularly the work 
Huete et al. (2013) on the interaction of detonation with density perturbations, by placing the focus on the mixture imperfections that add another type of convective perturbation (Griffond 2005, Farag et al. 2019), i.e., the local heat of combustion of the mixture. Results presented below demonstrate that the combination of the two sources of entropic perturbations derived from fuel-mass-fraction heterogeneities - i.e., perturbation of density and heat-release - may lead to order-of-magnitude differences in the turbulence generation rate when scaled with the fuel mass fraction variations. In addition, the acoustic energy, which typically exhibits a low-impact role in canonical shock-turbulence interactions, is of great importance here for weakly overdriven detonations.

The analysis makes use of the same mathematical description as that employed in the pioneering works of Ribner $(1954 a b, 1987)$ for shock waves and the posterior analyses in reactive shocks of Jackson et al. $(1990,1993)$, who assumed the ideal gas equation of state with constant specific heats and employed the fast-reaction limit to treat the detonation as a reactive shock. In addition to considering the effect of heat-release changes induced by the non-uniform upstream mixture, this work computes modified turbulent $\mathrm{RH}$ equations. It distinguishes a heterogeneous mixture from the corresponding uniform mixture with the same averaged upstream values (Lele 1992). As a result of the presence of heterogeneities in the fresh mixture, the averaged propagation speed of the detonation wave is modified, which also occurs with inert shocks (Hazak et al. 1998, Velikovich et al. 2012, Tian et al. 2020). Indeed, changes in the propagation speed have also been reported for detonations (Li et al. 2015, Mi et al. 2017a b. Prakash \& Raman 2019). The present work provides closed form analytical expressions in terms of the detonation properties and based on the burnt-gas dynamics to predict the averaged propagation speed.

The paper begins in Sec. 2 with the problem formulation for both base and linearly perturbed flow in the thin-detonation limit. Fourier analysis for two-dimensional and three-dimensional isotropic turbulent fields is employed to provide integral formulae for the amplification of the kinetic energy, vorticity, and density fluctuations in Sec. 3 . The near-field acoustic energy and the effect of the detonation passage in the turbulence scales are also analysed. The modification of the averaged $\mathrm{RH}$ conditions is obtained in Sec. 4 as well as the variation of the detonation propagation speed as a result of the upstream mixture heterogeneities. Final conclusions are presented in Sec. 5 The normal mode analysis, which is employed to describe the interaction of the planar detonation with a monochromatic pattern in the fuel mass fraction, is provided in appendix A

\section{Problem description}

\subsection{Upstream heterogeneous mixture}

The presence of non-equilibrium perturbations in the upstream gas coming from fuel mass fraction fluctuations must be accompanied with velocity perturbations to consistently satisfy the conservation equations. Similarly, pressure perturbations may be present in the heterogeneous mixture ahead of the shock. When the rotational, acoustic, entropic and composition perturbations result from the same physical mechanism, the corresponding spectra associated with the different types of perturbations are very similar. In particular, for stationary compressible isotropic turbulence (Holzer \& Siggia 1994, Miller 2000), the scalar energy spectra for the fuel mass fraction disturbances peak at the same wave number as the kinetic-energy dissipation spectrum, thereby suggesting the use of a similar spectrum form.

The relative influence of the different upstream perturbations on the downstream evolution of the flow depends on the initial perturbation amplitudes and the propagation 


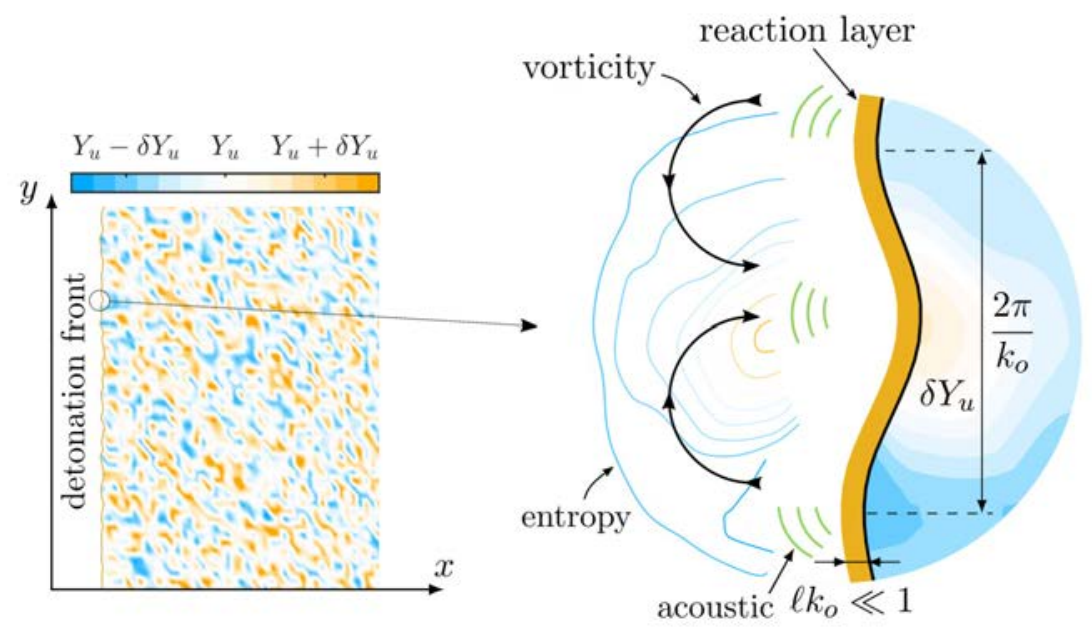

Figure 1: Sketch of the corrugated detonation front through a heterogeneous gaseous mixture, where the variation of the local fuel mass fraction satisfies $\left|\delta Y_{u}\right|=\left|Y_{u}-\left\langle Y_{u}\right\rangle\right| \ll$ 1 in order to apply linear theory. The detonation length $\ell$ is assumed to be considerably reduced than the characteristic upstream length $k_{o}^{-1}$.

Mach number. The latter dependence can be investigated using the linearized $\mathrm{RH}$ jump conditions, which show that the amplification factor affecting the mass fraction perturbations as they cross the front (with the corresponding changes in density and heat release) remains finite in the limit of large propagation Mach numbers $\mathcal{M}_{u} \gg 1$, whereas the corresponding amplification factors for the pressure and vorticity perturbations become proportional to $\mathcal{M}_{u}^{-2}$ and $\mathcal{M}_{u}^{-1}$, respectively. As result, in the strong shock limits, the upstream perturbations of vorticity and pressure play a secondary role with regards to the downstream flow. When dealing multi-component mixtures, the local specific-heat ratio $\gamma$ is also affected by mixture proportions, but this effect can be also neglected in the first approximation. Variations in $\gamma$ are expected to range between 1.1 and 1.4 between mixture compounds for light hydrocarbon fuels. Thus, order-of-unity variations in the fuel mass fraction provoke weak changes that are one order of magnitude smaller in the local value of $\gamma$. In addition, even admitting same-order variations in $\gamma$, these perturbations can be neglected for the same reason that velocity and pressure perturbations are not considered, namely they become proportional to $\mathcal{M}_{u}^{-2}$ in the strong shock limit.

In this work, a planar detonation front is considered to propagate through a heterogeneous gaseous mixture that is static and isobaric, so that the variations of the fuel mass fraction are only associated with perturbations in density and heat release, as sketched in Fig. 1. It is assumed that the deviation of the local fuel mass fraction $Y_{u}$ with respect to its average value $\left\langle Y_{u}\right\rangle \sim 1$ is sufficiently small for the linear theory to be applicable, $\left|\delta Y_{u}\right|=\left|Y_{u}-\left\langle Y_{u}\right\rangle\right| \ll 1$. For sufficiently weak perturbations, the isotropic heterogeneous mixture can be approximated by the linear superposition of independent modes, each one being characterized by a wavenumber $\vec{k}$, so that

$$
\delta Y_{u}\left(\vec{k}, \vec{x}_{u}\right)=\varepsilon(\vec{k}) e^{\mathrm{i} \vec{k} \cdot \vec{x}_{u}}
$$

where $\vec{x}_{u}$ is the vector defining the position in a reference frame at rest with respect to the upstream flow, and $\varepsilon(\vec{k})$ is the amplitude of the fuel mass fraction perturbations corresponding to a given wavenumber vector $\vec{k}$. 
(a)

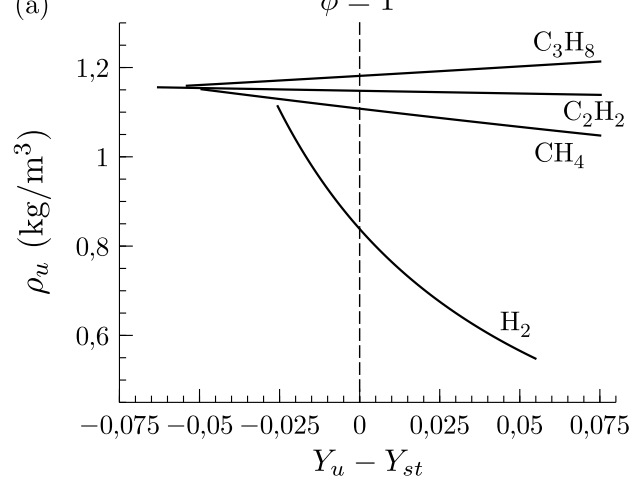

(b)

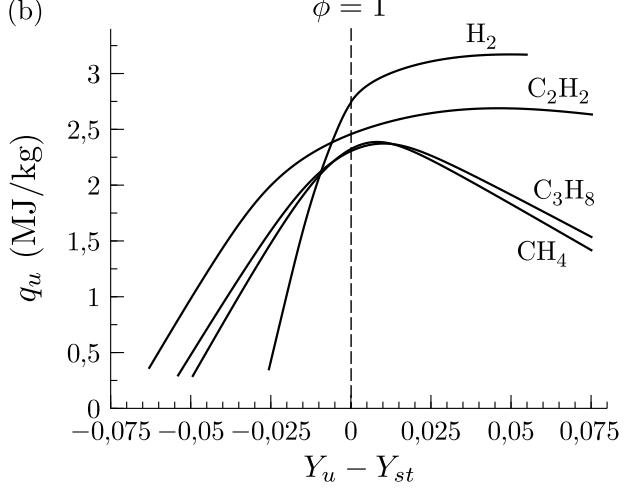

Figure 2: Variation of density (a) and heat release (b) with the fuel mass fraction for representative fuel-air mixtures. The dashed line represents the stoichiometric condition given by $Y_{u}=Y_{s t}(\phi=1)$. Computations are given for hydrogen, methane, acetylene and propane at $300 \mathrm{~K}$ and $10^{5} \mathrm{~Pa}$ in fresh conditions. The associated stoichiometric fuel mass fractions are $Y_{s t}=0.0285,0.0552,0.0705$ and 0.0603 , respectively.

Assuming that there is no privileged direction in the upstream flow, the spectrum of the fuel mass fraction perturbations is considered spatially homogeneous and isotropic. Thus, the wave-number vector $\vec{k}$ is uniformly distributed over the unit sphere (or around the unit semicircle in two dimensions). Therefore, the analysis consists of a superposition of linear perturbations whose amplitudes are exclusively functions of the wave-number amplitude $k=|\vec{k}|$. In such cases, the mean square of the fuel mass fraction perturbations in two-dimensional and three-dimensional geometries are

$$
\left\langle\delta Y_{u}^{2}\right\rangle_{2 \mathrm{D}}=\pi \int_{0}^{\infty} \varepsilon(k)^{2} k \mathrm{~d} k \text { and }\left\langle\delta Y_{u}^{2}\right\rangle_{3 \mathrm{D}}=4 \pi \int_{0}^{\infty} \varepsilon(k)^{2} k^{2} \mathrm{~d} k,
$$

respectively, where $\varepsilon(k)$ represents the spectrum of the fuel mass fraction. When fuel mass fraction perturbations are related to turbulent velocity fluctuations (Holzer \& Siggia 1994; Miller 2000), a plausible but arbitrary choice for the isotropic spectrum may be $\varepsilon(k)^{2} \sim$ $\left(k / k_{o}\right)^{2} /\left[1+\left(k / k_{o}\right)^{2}\right]^{17 / 6}$, where $k_{o}$ is the most representative wavenumber associated to the upstream turbulent flow (Batchelor 1953, Lee et al. 1993, Sagaut \& Cambon 2008, Livescu 2020). Compared to the detonation front, the fast-reaction model assumes that the detonation thickness is considerably smaller relative to the characteristic wavelength $\sim k_{o}^{-1}$ in the unimodal probability density distributions such that $k_{o} \ell \ll 1$ as displayed in Fig. 1. The two length scales are independent of each other. The characteristic scale of the spectrum depends on the type of mixing and turbulence upstream,whereas the detonation thickness mainly depends on the detonation properties through the reaction kinetics and temperature conditions. Although order-of-magnitude differences can be observed between different mixture properties, a rough estimate is $0.01-1$ centimetres as a characteristic scale for $\ell$, albeit upstream temperature and detonation overdrive can significantly change this value.

For a generic fuel-air mixture, if the fuel is heavier than air, positive values of $\delta Y_{u}$ are associated with positive upstream density perturbations, $\delta \rho_{u}>0$, and vice-versa. The sign of the perturbations of heat release can also be anticipated by taking into account the fact that heat release typically peaks under slightly rich conditions. Thus, positive values of $\delta Y_{u}$ lead to negative perturbations of heat release, $\delta q_{u}<0$, in sufficiently rich environments, while the opposite occurs for lean mixtures. 


\begin{tabular}{cccccccccc}
\hline \multirow{2}{*}{ Fuel } & \multirow{2}{*}{ Slopes } & \multicolumn{8}{c}{ Equivalence ratio $\phi$} \\
\cline { 3 - 10 } & & 0.25 & 0.5 & 0.75 & 1 & 1.25 & 1.5 & 1.75 & 2 \\
\hline \multirow{2}{*}{$\mathrm{H}_{2}$} & $W$ & -12.14 & -11.17 & -10.35 & -9.65 & -9.05 & -8.53 & -8.07 & -7.66 \\
& $H$ & 136.94 & 60.22 & 32.71 & 15.08 & 5.88 & 3.58 & 2.45 & 1.66 \\
\hline \multirow{2}{*}{$\mathrm{CH}_{4}$} & $W$ & -0.79 & -0.78 & -0.77 & -0.77 & -0.76 & -0.75 & -0.74 & -0.74 \\
& $H$ & 69.07 & 33.40 & 18.44 & 6.70 & -3.02 & -6.59 & -7.85 & -8.88 \\
\hline \multirow{2}{*}{$\mathrm{C}_{2} \mathrm{H}_{2}$} & $W$ & -0.11 & -0.11 & -0.11 & -0.11 & -0.11 & -0.11 & -0.11 & -0.11 \\
& $H$ & 52.91 & 23.21 & 9.36 & 4.77 & 2.50 & 1.08 & -0.03 & -0.8 \\
\hline \multirow{2}{*}{$\mathrm{C}_{3} \mathrm{H}_{8}$} & $W$ & 0.35 & 0.35 & 0.35 & 0.35 & 0.36 & 0.36 & 0.36 & 0.36 \\
& $H$ & 62.88 & 30.29 & 15.91 & 5.79 & -1.89 & -5.76 & -7.08 & -8.02 \\
\hline
\end{tabular}

Table 1: Dimensionless slopes of the density and heat release curves, $W$ and $H$, for $\mathrm{H}_{2}$, $\mathrm{CH}_{4}, \mathrm{C}_{2} \mathrm{H}_{2}$, and $\mathrm{C}_{3} \mathrm{H}_{8}$. The orange and blue background colours are applied for positive and negative values of the relation $H / W$, respectively.

The small parameter $\delta Y_{u}$ will be employed below to write the perturbed density and heat release fields as follows:

$$
\begin{aligned}
& \rho_{u}=\left\langle\rho_{u}\right\rangle+\delta \rho_{u}=\left\langle\rho_{u}\right\rangle\left(1+\delta Y_{u} W\right), \\
& q_{u}=\left\langle q_{u}\right\rangle+\delta q_{u}=\left\langle q_{u}\right\rangle\left(1+\delta Y_{u} H\right),
\end{aligned}
$$

where the parameters $W=\rho_{u}^{-1}\left(\mathrm{~d} \rho_{u} / \mathrm{d} Y_{u}\right)$ and $H=q_{u}^{-1}\left(\mathrm{~d} q_{u} / \mathrm{d} Y_{u}\right)$ relate to the local slopes of the $\rho_{u}\left(Y_{u}\right)$ and $q_{u}\left(Y_{u}\right)$ curves, respectively. For a "binary" fuel-air mixture with fuel and air mass fractions being $Y_{u}$ and $1-Y_{u}$, the dependence of the mixture density and the dimensionless function $W$ with the fuel mass fraction is noted as follows:

$$
\frac{\rho_{u}}{\rho_{a}}=\frac{1}{1-\left(1-\frac{W_{A}}{W_{F}}\right) Y_{u}} \quad \text { and } \quad W=\frac{1}{\rho_{u}} \frac{\mathrm{d} \rho_{u}}{\mathrm{~d} Y_{u}}=\frac{1-\frac{W_{A}}{W_{F}}}{1-\left(1-\frac{W_{A}}{W_{F}}\right) Y_{u}},
$$

where $\rho_{a}$ is the air density, and $W_{A}$ and $W_{F}$ refer to the air and fuel molecular weights, respectively.

The variation of the heat release with the fuel mass fraction cannot be accurately anticipated because it depends on the final composition of the burnt mixture. Detonations induce a strong compression work - through the irreversible transformation made by the shock - and a later expansion of the fluid, which makes the final temperature differ from the adiabatic flame temperature of isobaric combustion. It is widely known however that heat release peaks close to stoichiometric conditions for slightly rich mixtures, a property that can be used to anticipate the sign of the slope of the heat release-fuel mass fraction curve. Accurate values of $q_{u}$ can be obtained with either numerical codes or experimental data. Figure 2 has been computed with an in-house thermochemical code validated with NASA-CEA (Gordon \& McBride 1994) and CANTERA (Goodwin et al. 2018), including Caltech's Shock and Detonation Toolbox (Browne et al. 2008). Here, the variation of density (a) and heat release (b) with the fuel mass fraction is shown for different gases, including hydrogen $\left(\mathrm{H}_{2}\right)$, methane $\left(\mathrm{CH}_{4}\right)$, acetylene $\left(\mathrm{C}_{2} \mathrm{H}_{2}\right)$ and propane $\left(\mathrm{C}_{3} \mathrm{H}_{8}\right)$, with stoichiometric fuel mass fractions $Y_{s t}=0.0285,0.0552,0.0705$ and 0.0603 , respectively. Representative values of $W$ and $H$ obtained from these computations are 
given in Table 1 for these fuels and different equivalence ratios ranging from very lean to moderately rich mixtures.

\subsection{Homogeneous Rankine-Hugoniot equations}

Neglecting the deviation from the mean values, the flow variables in the burnt gas are obtained by integrating the conservation equations in the streamwise direction, yielding

$$
\begin{aligned}
\rho_{u} u_{u} & =\rho_{d} u_{d}, \\
p_{u}+\rho_{u} u_{u}^{2} & =p_{d}+\rho_{d} u_{d}^{2}, \\
h_{u}+\frac{1}{2} u_{u}^{2} & =h_{d}+\frac{1}{2} u_{d}^{2},
\end{aligned}
$$

for the mass, streamwise momentum, and energy conservation equations, respectively. Here, the subscripts $u$ and $d$ refer to the upstream and downstream dimensional flow properties, respectively, including the velocity $u$, density $\rho$ (or specific volume $V=\rho^{-1}$ ), pressure $p$, and enthalpy $h$. The upstream flow comprises a mixture of gases, each of which is modelled as a calorically perfect gas, i.e., the specific heats, and therefore the adiabatic index $\gamma$ are assumed to be constant throughout the shock compression and combustion processes. Thus, the enthalpy variation is written as a function of a single value of $\gamma$

$$
h_{d}-h_{u}=q_{u}+\frac{\gamma}{\gamma-1}\left(\frac{p_{d}}{\rho_{d}}-\frac{p_{u}}{\rho_{u}}\right),
$$

where $q_{u}$ represents the heat released in the reaction front per unit mass of mixture. In realistic conditions, the value of $\gamma$ depends on both mixture composition and temperature, which experience significant changes across the detonation wave. In qualitative terms, when the effect of temperature dominates the variation of $\gamma$ by the excitation of vibrational modes, the specific heat ratio is expected to monotonically decrease with temperature. However, a change in the structure of the molecules exists as a result of the combustion process, the total variation of $\gamma$ must include information of the initial and final composition of the mixture. However, since upstream thermal energy can be neglected in the first approximation, the value used in the computations should be the value associated with the burnt gas conditions to minimize the error when $\gamma$ is constant.

Algebraic manipulation of 2.6a)-2.7 provides the jump equations across the reaction front, namely,

$$
\begin{aligned}
\mathcal{R}_{d}=\frac{\rho_{d}}{\rho_{u}} & =\frac{(\gamma+1) \mathcal{M}_{u}^{2}}{\gamma \mathcal{M}_{u}^{2}+1-\left[\left(\mathcal{M}_{u}^{2}-1\right)^{2}-4 Q \mathcal{M}_{u}^{2}\right]^{1 / 2}} \\
\mathcal{P}_{d}=\frac{p_{d}}{p_{u}} & =\frac{\gamma \mathcal{M}_{u}^{2}+1+\gamma\left[\left(\mathcal{M}_{u}^{2}-1\right)^{2}-4 Q \mathcal{M}_{u}^{2}\right]^{1 / 2}}{\gamma+1} \\
& =\frac{(\gamma+1) \mathcal{R}_{d}-\gamma+1+4 \mathcal{R}_{d} Q \gamma /(\gamma+1)}{\gamma+1-(\gamma-1) \mathcal{R}_{d}}
\end{aligned}
$$

for density and pressure, respectively, while the Mach number of the burnt-gas flow is

$$
\mathcal{M}_{d}=\frac{u_{d}}{a_{d}}=\left\{\frac{\gamma \mathcal{M}_{u}^{2}+1-\left[\left(\mathcal{M}_{u}^{2}-1\right)^{2}-4 Q \mathcal{M}_{u}^{2}\right]^{1 / 2}}{\gamma \mathcal{M}_{u}^{2}+1+\gamma\left[\left(\mathcal{M}_{u}^{2}-1\right)^{2}-4 Q \mathcal{M}_{u}^{2}\right]^{1 / 2}}\right\}^{1 / 2}
$$


where $a_{d}$ denotes the speed of sound and

$$
Q=\frac{\left(\gamma^{2}-1\right)}{2 a_{u}^{2}} q_{u}
$$

is the dimensionless heat release per unit mass.

The so-called Chapman-Jouguet (CJ) condition, which is given by $\left(\mathcal{M}_{u}^{2}-1\right)^{2}-$ $4 Q \mathcal{M}_{u}^{2}=0$, defines the minimum detonation strength. Thus, the maximum downstream Mach number is associated with a given energy release as follows:

$$
\mathcal{M}_{\mathrm{cj}}=(1+Q)^{1 / 2}+Q^{1 / 2} .
$$

This value is related to the maximum flow expansion of the hot gases in the reaction layer. Within this subsonic region behind the shock, the flow is expanded up to sonic conditions, $\mathcal{M}_{d}=1$, corresponding to the well-known Chapman-Jouguet condition for detonation waves. As a result, the detonation wave decouples from downstream influences, and its motion is self-sustained by the expansion of the products resulting from the exothermic transformation.

\subsection{Linear interaction analysis}

As illustrated in Fig. 2 and Table 1, deviations in the fuel mass fraction are often accompanied by perturbations in density and/or heat-release. Depending on the particular conditions, i.e., type of fuel and average equivalence ratio of the unburnt mixture, the dimensionless slope $H$ may reach values of order 10 or greater. It also occurs with the function $W$ in hydrogen-air mixtures (or any other fuel much heavier than air not shown in the table). This finding suggests that $\delta Y_{u}$ may not be a good reference scale for the upstream perturbation field given that linear (small) variations in the fuel mass fraction may be associated with non-linear (large) perturbations in $\rho_{u}$ and/or $q_{u}$. For any flow condition, to ensure that small changes $\delta Y_{u}$ do not lead to large input perturbations into the system, the small parameter should simply be redefined in the form $\epsilon(k)=\varepsilon(k) \sqrt{W^{2}+H^{2}}$, and $\epsilon(k) \ll 1$ is a requirement. Thus, even if $W$ or $H$ are large compared to unity, the perturbations in the fuel mass fraction will be sufficiently small for all flow perturbations to remain within the limits of linear theory. Similarly, larger fuel mass fraction perturbations will be permitted for $W \sim H \ll 1$.

When written in a reference frame moving with the detonation front, $x_{l}=u_{u}$, the upstream density and heat-release perturbations can be written as follows:

$$
\hat{\rho}_{u}=\frac{\delta \rho_{u}}{\left\langle\rho_{u}\right\rangle}=\epsilon(k) \frac{W}{\sqrt{W^{2}+H^{2}}} e^{\mathrm{i}\left(k_{x} u_{u} t+k_{y} y+k_{z} z\right)}
$$

and

$$
\hat{q}_{u}=\frac{\delta q_{u}}{\left\langle q_{u}\right\rangle}=\epsilon(k) \frac{H}{\sqrt{W^{2}+H^{2}}} e^{\mathrm{i}\left(k_{x} u_{u} t+k_{y} y+k_{z} z\right)},
$$

where use is composed of (2.1), (2.3) and (2.4) and the above definition of $\epsilon(k)$. The pre-exponential factors $W / \sqrt{W^{2}+H^{2}}$ and $H / \sqrt{W^{2}+H^{2}}$ represent the relative effects on density and heat-release fluctuations of a small change of the fuel mass fraction. A closer inspection of the RH jump relationships shows that positive values of $\delta \rho_{u}$ and $\delta q_{u}$ result in higher downstream pressures. In terms of fuel mass fraction perturbation $\delta Y_{u}$, constructive effects are to be expected in lean heavy-fuel or rich light-fuel mixtures, while destructive contributions will occur in lean light-fuel or rich heavy-fuel mixtures.

Using the modulus of the wavenumber vector $k$ and its product with the speed of sound in the burnt gas $a_{d} k$ as characteristic spatial and temporal frequencies, the 
spatio-temporal variables can be written in non-dimensional form as $(\hat{x}, \hat{y}, \hat{z})=k \vec{x}$ and $\tau=a_{d} k t$, where $\vec{x}$ is measured in the burnt gas reference frame. However, it is always possible to simplify the formulation by rotating the reference frame around the direction of propagation of the detonation such that only two spatial coordinates remain in the problem: one in the axis of the detonation propagation, $\hat{x}$, and the other perpendicular to it at any arbitrary direction, $\hat{y}$.

The linear perturbations in the burnt gas are defined with functions of order unity (denoted with hat symbol) and scaled with the small parameter $\epsilon$, namely

$$
\begin{aligned}
& p(x, y, \tau)=\rho_{d} a_{d}^{2}\left[\gamma^{-1}+\epsilon \hat{p}(\hat{x}, \hat{y}, \tau)\right], \\
& \rho(x, y, \tau)=\rho_{d}[1+\epsilon \hat{\rho}(\hat{x}, \hat{y}, \tau)], \\
& u(x, y, \tau)=a_{d}\left[\mathcal{M}_{d}\left(\mathcal{R}_{d}-1\right)+\epsilon \hat{u}(\hat{x}, \hat{y}, \tau)\right], \\
& v(x, y, \tau)=a_{d} \epsilon \hat{v}(\hat{x}, \hat{y}, \tau),
\end{aligned}
$$

which are introduced in the Euler equations to give

$$
\begin{aligned}
& \frac{\partial \hat{\rho}}{\partial \tau}=-\frac{\partial \hat{u}}{\partial \hat{x}}-\frac{\partial \hat{v}}{\partial \hat{y}}, \\
& \frac{\partial \hat{u}}{\partial \tau}=-\frac{\partial \hat{p}}{\partial \hat{x}}, \\
& \frac{\partial \hat{v}}{\partial \tau}=-\frac{\partial \hat{p}}{\partial \hat{y}}, \\
& \frac{\partial \hat{\rho}}{\partial \tau}=\frac{\partial \hat{p}}{\partial \tau} .
\end{aligned}
$$

The system of linear equations must supplemented by the boundary conditions associated to isolated detonation. As the burnt-gas flow field reduces to linear gas dynamics within the domain contained between $\hat{x}=-\tau+c_{t}$ (first left-traveling acoustic wave) and $\hat{x}=\mathcal{M}_{d} \tau+c_{t}$ (detonation front position) in the $\hat{x}-\tau$ plane. The arbitrary constant $c_{t}$, which is defined with the temporal reference frame, does not play any role in the long-time asymptotic analysis.

For the sake of simplicity, the isolated assumption is considered in this work, which translates into the omission of front-traveling waves reaching the detonation wave from behind. As a direct consequence, the detonation is only excited with the monochromatic perturbation of the upstream flow, thereby resulting in a single oscillation frequency $\omega_{d}=\left(k_{x} / k\right)\left(u_{u} / a_{d}\right)=\kappa_{x} \mathcal{R}_{d} \mathcal{M}_{d}$, where $\kappa_{i}=k_{i} / k$ for $i=\{x, y, z\}$ is the dimensionless wave number component of the upstream flow. Linearized RH relationships, which involve the perturbation of the detonation position with respect to its planar form $\xi_{d}=k\left[x_{l, s}(t)-u_{u} t\right]$

$$
\begin{aligned}
\frac{\mathcal{R}_{d}-1}{\mathcal{R}_{d}} \frac{\mathrm{d} \xi_{d}}{\mathrm{~d} \tau} & =\frac{1-\Gamma}{2 \mathcal{M}_{d}} \hat{p}_{d}-\frac{\mathcal{M}_{d}\left(\mathcal{R}_{d}-2+\Delta\right) W-\mathcal{M}_{d}(1-\Delta) H}{2 \sqrt{W^{2}+H^{2}}} e^{-\mathrm{i} \omega_{d} \tau} e^{\mathrm{i} \kappa_{y} \hat{y}}, \\
\hat{u}_{d} & =\frac{1+\Gamma}{2 \mathcal{M}_{d}} \hat{p}_{d}-\frac{\mathcal{M}_{d}\left(\mathcal{R}_{d}-\Delta\right) W+\mathcal{M}_{d}(1-\Delta) H}{2 \sqrt{W^{2}+H^{2}}} e^{-\mathrm{i} \omega_{d} \tau} e^{\mathrm{i} \kappa_{y} \hat{y}}, \\
\hat{\rho}_{d} & =\frac{\Gamma}{\mathcal{M}_{d}^{2}} \hat{p}_{d}+\frac{\Delta W-(1-\Delta) H}{\sqrt{W^{2}+H^{2}}} e^{-\mathrm{i} \omega_{d} \tau} e^{\mathrm{i} \kappa_{y} \hat{y}}, \\
\hat{v}_{d} & =-\mathcal{M}_{d}\left(\mathcal{R}_{d}-1\right) \frac{\partial \xi_{d}}{\partial \hat{y}}
\end{aligned}
$$

are employed to calculate the amplitudes accompanying the factor $e^{-\mathrm{i} \omega_{d} \tau} e^{\mathrm{i} \kappa_{y} \hat{y}}$ in the 
non-dimensional perturbations. The quantity

$$
\Gamma=\left.\frac{p_{d}-p_{u}}{V_{u}-V_{d}} \frac{\mathrm{d} V}{\mathrm{~d} p}\right|_{d}=\frac{\gamma \mathcal{M}_{u}^{2}}{\mathcal{R}_{d}^{2}}\left(\frac{\partial \mathcal{P}_{d}}{\partial \mathcal{R}_{d}}\right)^{-1}
$$

measures the slope of the $\mathrm{RH}$ curve relative to the Rayleigh-Mickelson line, and the term

$$
\Delta=\frac{1+Q \mathcal{R}_{d}\left(\frac{\gamma-1}{\gamma+1}\right)}{1+Q}<1
$$

accounts for the variation of the post-detonation state due to the upstream changes in density and heat release.

Notice that the linear perturbation variables admit the breakdown in terms of potential (acoustic) and entropic-rotational contributions (Kovasznay 1953. Chu \& Kovásznay 1958). The former takes the form of traveling waves, while the latter remains steady in the reference frame moving with the burnt-gas particles in absence of diffusive effects. This property is exploited in the normal mode analysis performed in the appendix A, where the transfer functions describing the amplitude of the burnt-gas perturbation modes as a function of the detonation properties is described.

\section{Detonation-induced turbulence}

This section is devoted to the interaction of a planar detonation wave with a $2 \mathrm{D} / 3 \mathrm{D}$ isotropic $2 \mathrm{D} / 3 \mathrm{D}$ field of fuel-mass-fraction fluctuation. In the previous section, it was shown that the pre-shock field can be characterized by the wave number vector $\vec{k}=$ $\left(k_{x}, k_{y}\right)=k(\cos \theta, \sin \theta)$ in a reduced $2 \mathrm{D}$ geometry, i.e., a planar transverse wave with wavenumber $\vec{k}$ that intersects a planar detonation wave with an incidence angle $\theta$. In two dimensions, isotropy translates into considering situations where $k$ is uniformly distributed in the range $0 \leqslant \theta \leqslant \pi$. In three dimensions, it is assumed that $k$ is uniformly distributed over the unit sphere. Linear theory admits the computation of the full spectrum by direct superposition of small perturbations, in which the amplitudes are an exclusive function of $k$ with the different modes being uncorrelated.

\subsection{Turbulent kinetic energy}

An important feature that deserves particular attention is the turbulence kinetic energy (TKE) generated by the wrinkled detonation front. When scaled with the square of the sound speed in the burnt gas, it can be expressed as

$$
\mathrm{TKE}_{2 \mathrm{D}}=\frac{1}{2} \frac{\left\langle\delta v_{i} \delta v_{i}\right\rangle}{a_{d}^{2}}=\frac{1}{2}\left\langle\hat{u}^{2}+\hat{v}^{2}\right\rangle_{2 \mathrm{D}} \int_{0}^{\infty} \epsilon(k)^{2} k \mathrm{~d} k=\frac{1}{2}\left\langle\hat{u}^{2}+\hat{v}^{2}\right\rangle_{2 \mathrm{D}}\left\langle\delta Y_{u}^{2}\right\rangle_{2 \mathrm{D}}
$$

with

$$
\left\langle\hat{u}^{2}+\hat{v}^{2}\right\rangle_{2 \mathrm{D}}=\frac{2}{\pi} \int_{0}^{\pi / 2}\left(|\hat{u}|^{2}+|\hat{v}|^{2}\right) \mathrm{d} \theta
$$

provided $\left\langle\delta Y_{u}^{2}\right\rangle_{2 \mathrm{D}}$ is known from 2.2 . Thanks to the isotropy condition of the perturbation field, the influence of the shape of the spectrum enters as an integral contribution that can be factored out. Equivalent three dimensional computations can be performed by defining

$$
\left\langle\hat{u}^{2}+\hat{v}^{2}\right\rangle_{3 \mathrm{D}}=\int_{0}^{\pi / 2}\left(|\hat{u}|^{2}+|\hat{v}|^{2}\right) \sin \theta \mathrm{d} \theta
$$




$$
\mathcal{M}_{u} / \mathcal{M}_{\mathrm{cj}}-1
$$
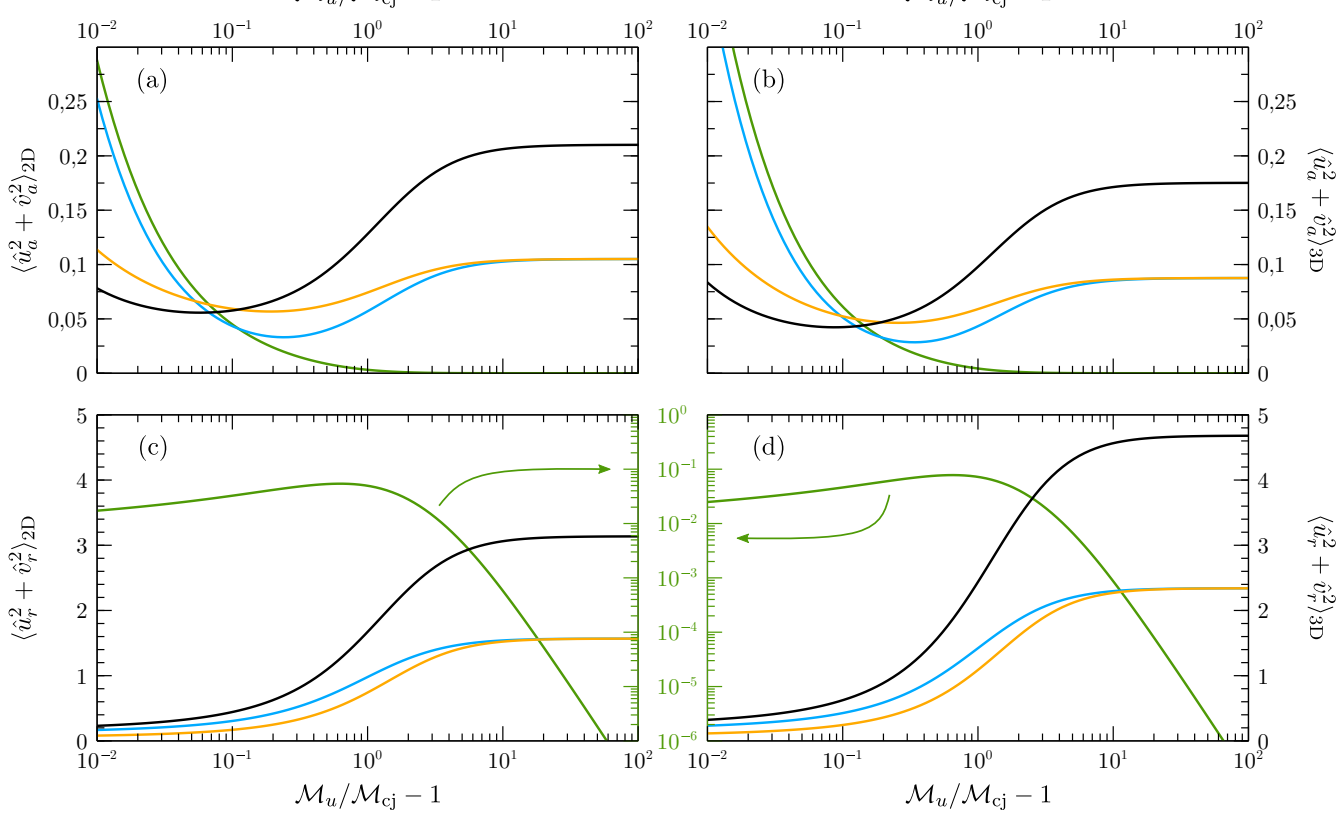

Figure 3: Acoustic (a-b) and rotational (c-d) contributions to the turbulent kinetic energy in 2D ( $\mathrm{a}$ and $\mathrm{c}$ ) and $3 \mathrm{D}(\mathrm{b}$ and $\mathrm{d}$ ) geometries. Computations are shown as a function of the overdrive parameter $\mathcal{M}_{u} / \mathcal{M}_{\mathrm{cj}}-1$ for $\gamma=1.2, Q=1$, and $|W| \gg|H|(-)$, $|H| \gg|W|(\longleftarrow), W=H(\longleftarrow)$ and $W=-H(-)$.

where $\hat{u}$ and $\hat{v}$ are the streamwise and transverse velocity components. The normalization criterion applied is that $\left\langle\hat{u}^{2}+\hat{v}^{2}\right\rangle_{2 \mathrm{D}, 3 \mathrm{D}}$ must be equal to unity when the integrand function is spatially uniform and equal to unity.

In the far-field asymptotic regime, the kinetic energy can be conveniently computed by accounting for the separate contributions of the rotational and acoustic velocity fields $\left\langle\hat{u}^{2}+\hat{v}^{2}\right\rangle=\left\langle\hat{u}_{r}^{2}+\hat{v}_{r}^{2}\right\rangle+\left\langle\hat{u}_{a}^{2}+\hat{v}_{a}^{2}\right\rangle$, to give

$$
\begin{aligned}
& \left\langle\hat{u}^{2}+\hat{v}^{2}\right\rangle_{2 \mathrm{D}}=\frac{2}{\pi} \int_{0}^{\pi / 2}\left(\mathbb{U}_{r}^{2}+\mathbb{V}_{r}^{2}\right) \mathrm{d} \theta+\frac{2}{\pi} \int_{0}^{\theta_{c}}\left(\mathbb{U}_{a}^{2}+\mathbb{V}_{a}^{2}\right) \mathrm{d} \theta, \\
& \left\langle\hat{u}^{2}+\hat{v}^{2}\right\rangle_{3 \mathrm{D}}=\int_{0}^{\pi / 2}\left(\mathbb{U}_{r}^{2}+\mathbb{V}_{r}^{2}\right) \sin \theta \mathrm{d} \theta+\int_{0}^{\theta_{c}}\left(\mathbb{U}_{a}^{2}+\mathbb{V}_{a}^{2}\right) \sin \theta \mathrm{d} \theta,
\end{aligned}
$$

where the amplitudes $\mathbb{U}_{r}, \mathbb{V}_{r}, \mathbb{U}_{a}$, and $\mathbb{V}_{a}$, provided in appendix $\mathrm{A}$ are explicit functions of $\theta=\tan ^{-1}\left(k_{y} / k_{x}\right)$ and the remaining governing parameters: $\gamma, \mathcal{M}_{u}, Q, W$, and $H$. To compute the rotational contribution one must separate the high-frequency and lowfrequency amplitudes, as given in $(\mathrm{A} 29$, which must be integrated over the two different domains $\int_{0}^{\theta_{c}}(\ldots) \mathrm{d} \theta$ and $\int_{\theta_{c}}^{\pi / 2}(\ldots) \mathrm{d} \theta$, respectively.

Figure 3 shows the acoustic (a-b) and rotational (c-d) contributions to the total kinetic energy in both two-dimensional ( $\mathrm{a}$ and $\mathrm{c}$ ) and three-dimensional ( $\mathrm{b}$ and $\mathrm{d}$ ) isotropic configurations. The averages are plotted as a function of the overdrive parameter $\mathcal{M}_{u} / \mathcal{M}_{\mathrm{cj}}-1$. The correlation between $W$ and $H$ has a strong impact on the generation of turbulent kinetic energy. For example, heat-release variations, either for $|H| \gg|W|$ or $H=-W$, produce higher levels of acoustic energy when the overdrive is small, a finding that can be anticipated with the normal mode analysis, as shown in Fig. 14 in appendix 

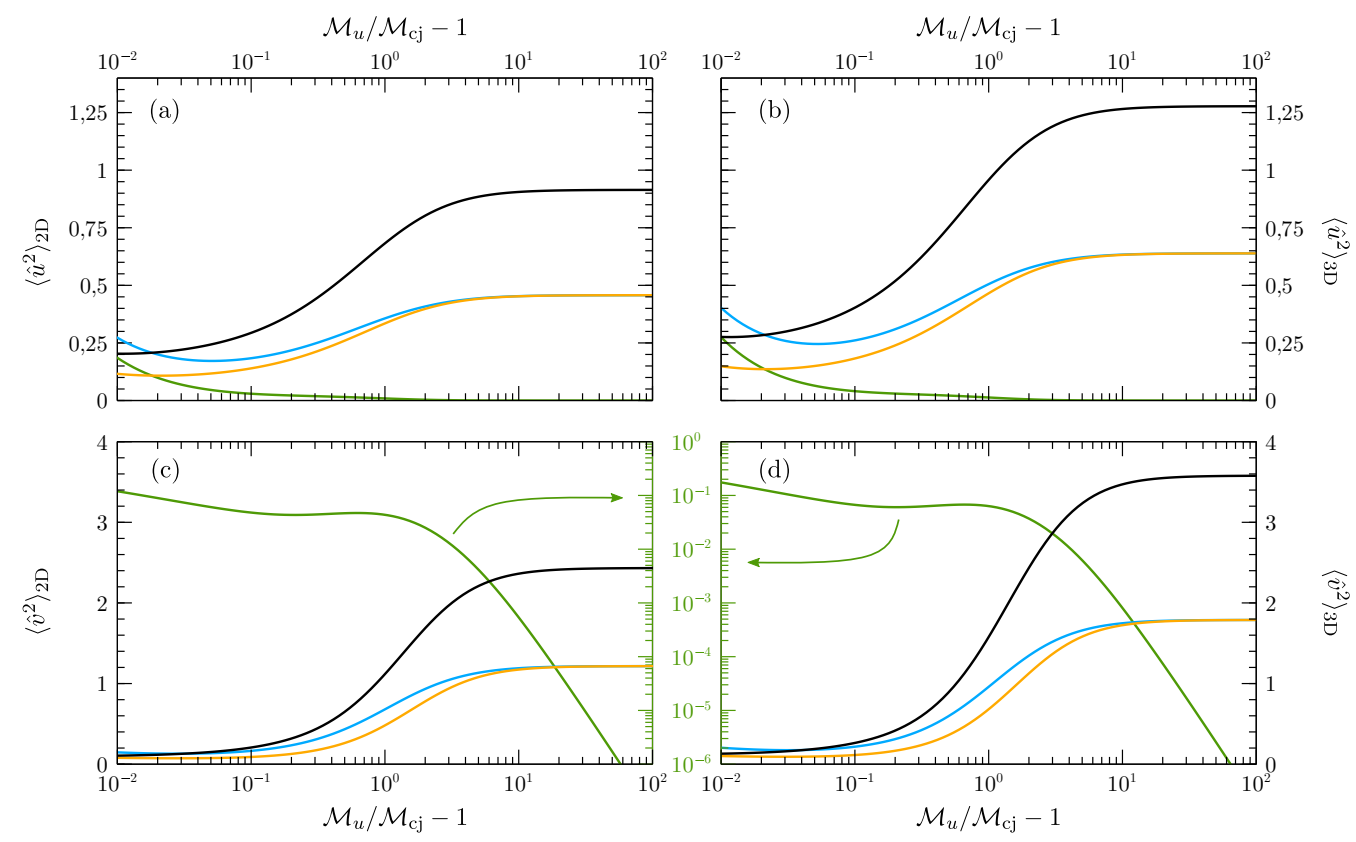

Figure 4: Longitudinal (a-b) and transverse (c-d) contributions to the turbulent kinetic energy in 2D ( $\mathrm{a}$ and $\mathrm{c}$ ) and 3D ( $\mathrm{b}$ and $\mathrm{d}$ ) geometries. Computations are shown as a function of the overdrive parameter $\mathcal{M}_{u} / \mathcal{M}_{\mathrm{cj}}-1$ for $\gamma=1.2, Q=1$, and $|W| \gg|H|$ $(-),|H| \gg|W|(-), W=H(-)$ and $W=-H(-)$.

A The unbounded growth of the acoustic perturbations in the CJ condition warns about the limits of validity of the linear thin-detonation model, which gains in reliability as the overdrive increases.

The cases selected for the calculations and the associated colour code will be maintained in the following figures. In all the plots, the black curves correspond to the reference case where the upstream perturbation field is dominated by density changes, as in Huete et al. (2013). In this case, the average fuel mass fraction is close to the peak of heat release, which leads to a negligible contribution of heat-release variations with the fuel mass fraction. As an opposite limit, the green curves represent cases where heat-release variations dominate over density changes, which is applicable to mixtures of gases with similar density and located far-off the heat release peak. The other two distinguished cases refer to positive (orange) and negative (blue) correlations of density and heat release perturbations. The former is applicable to light fuel-air mixtures $\left(W_{F}<W_{A}\right)$ in sufficiently rich conditions or heavy fuel-air mixtures $\left(W_{F}>W_{A}\right)$ in sufficiently lean conditions. On the other hand, negative correlations are applicable to light fuelair mixtures in sufficiently lean conditions or heavy fuel-air mixtures in sufficiently rich conditions. Four examples are given in Table 1 where the orange and blue background colours denote the mentioned positive and negative correlations, respectively.

Regarding the rotational contribution, the degree of overdrive does not modify the hierarchy of effects associated to the correlation between $H$ and $W$ for one single frequency, as displayed in Fig. 15 in appendix A. Notice that the case $|H| \gg|W|$ (green lines) uses its own log-log scale, which is shown between subplots (c) and (d), because it would have been impossible to distinguish it from the horizontal axis otherwise. With this consideration in mind, it is seen that the case with exclusively density perturbations yields 
the highest intensity of turbulence generation, and the effect of heat release is to cushion the kinetic energy deposited in form of eddies behind the detonation wave. The results also show that the rotational contribution dominates the generation of turbulent kinetic energy over the acoustic field by one order of magnitude, except for weakly overdriven detonations, when both become the same order. Some features in Fig. 3 are similar for the acoustic and rotational contributions. For example, the qualitative picture of twodimensional and three-dimensional cases is very similar. In addition, density disturbances dominate for finite to high degrees of overdrives. Thus, the turbulent kinetic energy generated for $|H| \gg|W|$ is much smaller and tends to zero with the overdrive, while the cases $H=-W$ and $H=W$ render similar values for strongly overdriven detonations, an asymptotic value that corresponds to the half of that obtained for $|W| \gg|H|$. The latter is easily deduced from $(2.13)$ since the relative amplitude of the density field squared, $W^{2} /\left(W^{2}+H^{2}\right)$, is unity for $|W| \gg|H|$ and $1 / 2$ for $H= \pm W$, while the relative amplitude of the heat release field squared, $H^{2} /\left(W^{2}+H^{2}\right)$, vanishes for $|W| \gg|H|$.

The breakdown of longitudinal and transverse contributions of the kinetic energy can be computed separately as

$$
\begin{aligned}
& \left\langle\hat{u}^{2}\right\rangle_{2 \mathrm{D}}=\left\langle\hat{u}_{r}^{2}\right\rangle_{2 \mathrm{D}}+\left\langle\hat{u}_{a}^{2}\right\rangle_{2 \mathrm{D}}=\frac{2}{\pi} \int_{0}^{\pi / 2} \mathrm{U}_{r}^{2} \mathrm{~d} \theta+\frac{2}{\pi} \int_{0}^{\theta_{c}} \mathbb{U}_{a}^{2} \mathrm{~d} \theta \\
& \left\langle\hat{u}^{2}\right\rangle_{3 \mathrm{D}}=\left\langle\hat{u}_{r}^{2}\right\rangle_{3 \mathrm{D}}+\left\langle\hat{u}_{a}^{2}\right\rangle_{3 \mathrm{D}}=\int_{0}^{\pi / 2} \mathbb{U}_{r}^{2} \sin \theta \mathrm{d} \theta+\int_{0}^{\theta_{c}} \mathbb{U}_{a}^{2} \sin \theta \mathrm{d} \theta
\end{aligned}
$$

and

$$
\begin{aligned}
& \left\langle\hat{v}^{2}\right\rangle_{2 \mathrm{D}}=\left\langle\hat{v}_{r}^{2}\right\rangle_{2 \mathrm{D}}+\left\langle\hat{v}_{a}^{2}\right\rangle_{2 \mathrm{D}}=\frac{2}{\pi} \int_{0}^{\pi / 2} \mathbb{V}_{r}^{2} \mathrm{~d} \theta+\frac{2}{\pi} \int_{0}^{\theta_{c}} \mathbb{V}_{a}^{2} \mathrm{~d} \theta \\
& \left\langle\hat{v}^{2}\right\rangle_{3 \mathrm{D}}=\left\langle\hat{v}_{r}^{2}\right\rangle_{3 \mathrm{D}}+\left\langle\hat{v}_{a}^{2}\right\rangle_{3 \mathrm{D}}=\int_{0}^{\pi / 2} \mathbb{V}_{r}^{2} \sin \theta \mathrm{d} \theta+\int_{0}^{\theta_{c}} \mathbb{V}_{a}^{2} \sin \theta \mathrm{d} \theta
\end{aligned}
$$

respectively, where $\left\langle\hat{u}^{2}+\hat{v}^{2}\right\rangle_{2 \mathrm{D}, 3 \mathrm{D}}=\left\langle\hat{u}^{2}\right\rangle_{2 \mathrm{D}, 3 \mathrm{D}}+\left\langle\hat{v}^{2}\right\rangle_{2 \mathrm{D}, 3 \mathrm{D}}$ for both rotational and acoustic contributions, independently.

Figure 4 shows the average streamwise (a-b) and transverse (c-d) contributions to the total kinetic energy in both two-dimensional (a and c) and three-dimensional (b and d) isotropic flows. The averages are plotted as a function of the overdrive parameter $\mathcal{M}_{u} / \mathcal{M}_{\text {cj }}-1$. In consonance with Fig. 15, the lateral component is higher than the longitudinal part. As expected, the strong-shock limit is governed by the density-induced turbulence in both longitudinal and transverse contributions. In the case $|H| \gg|W|$, shown on its own log-log scale, the turbulence generation is very weak over most of the overdrive domain. For weakly overdriven detonations, however, the effect of heat-release variations has a non-negligible impact in the generation of turbulence, which, as observed in Fig. 3, is mainly derived from the acoustic contribution. In agreement with the previous plot, for three-dimensional random isotropic fields, the generation of turbulence is more intense for both lateral and streamwise components, and negative correlations render higher levels of turbulence. Thus, when the fuel mass fraction is outside the heat-release peak, turbulence generation is expected to be enhanced from the hydrodynamical point of view in light fuel-air mixtures in sufficiently lean conditions or heavy fuel-air mixtures in sufficiently rich conditions. 

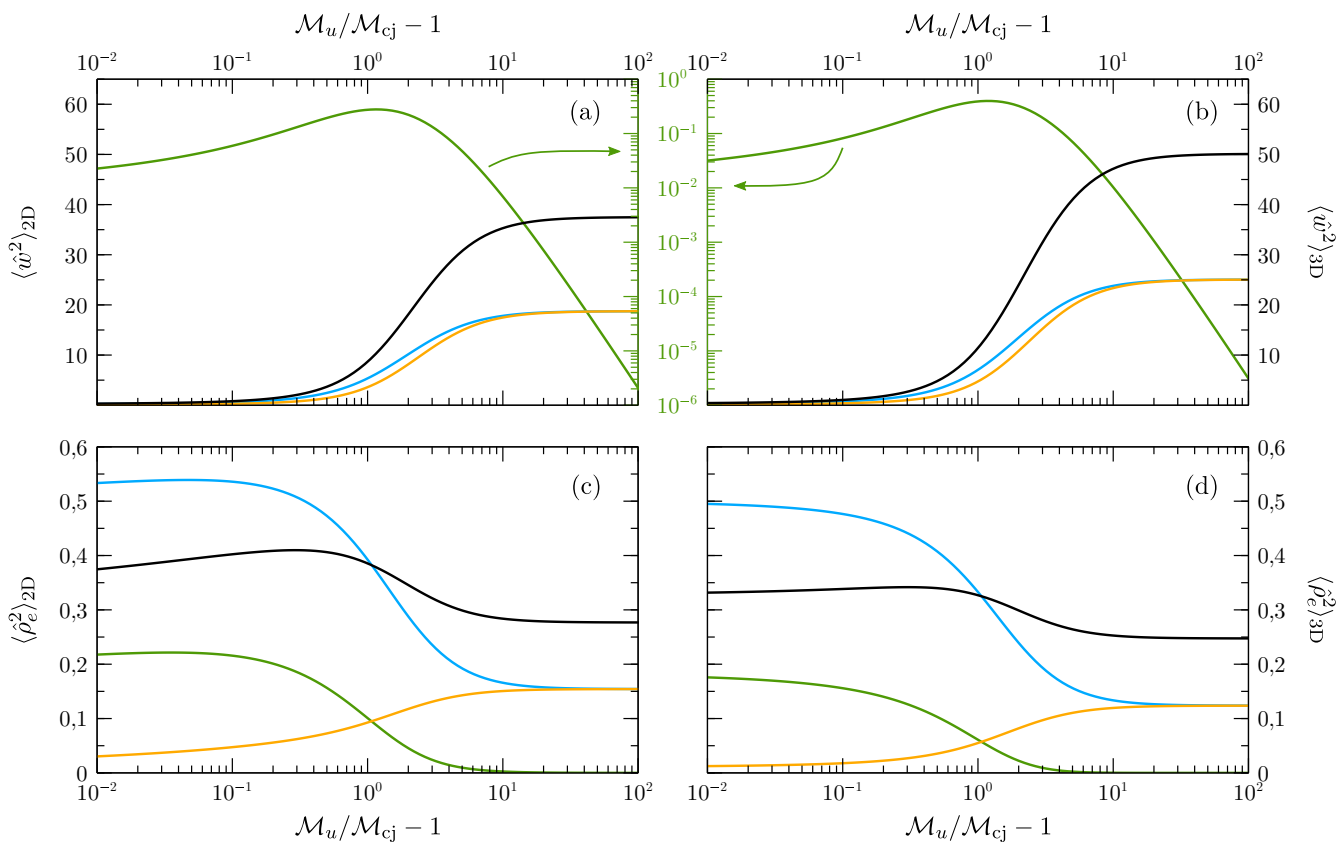

Figure 5: Averaged square vorticity (a-b) and square entropic density (c-d) in 2D (a and c) and $3 \mathrm{D}(\mathrm{b}$ and $\mathrm{d})$ geometries. Computations are shown as a function of the overdrive parameter $\mathcal{M}_{u} / \mathcal{M}_{\mathrm{cj}}-1$ for $\gamma=1.2, Q=1$, and $|W| \gg|H|(-),|H| \gg|W|(-)$, $W=H(-)$ and $W=-H(-)$.

\subsection{Enstrophy and averaged pressure and density fields}

The average rotational motion behind the detonation can be measured by the so-called enstrophy that takes the form

$$
\left\langle\Omega^{2} \kappa_{y}^{2}\right\rangle_{2 \mathrm{D}}=\frac{2}{\pi} \int_{0}^{\pi / 2} \mathrm{O}^{2} \sin ^{2} \theta \mathrm{d} \theta, \quad\left\langle\Omega^{2} \kappa_{y}^{2}\right\rangle_{3 \mathrm{D}}=\int_{0}^{\pi / 2} \mathrm{O}^{2} \sin ^{3} \theta \mathrm{d} \theta,
$$

where $\mathbb{O}$ must be evaluated from $\mathrm{A} 25$ in appendix $\mathrm{A}$ and $\kappa_{y}=\sin \theta$. By $\operatorname{similar}$ arguments, the averaged values of the squared density perturbations are

$$
\begin{aligned}
& \left\langle\hat{\rho}^{2}\right\rangle_{2 \mathrm{D}}=\left\langle\hat{\rho}_{e}^{2}\right\rangle_{2 \mathrm{D}}+\left\langle\hat{\rho}_{a}^{2}\right\rangle_{2 \mathrm{D}}=\frac{2}{\pi} \int_{0}^{\pi / 2} \mathrm{D}_{e}^{2} \mathrm{~d} \theta+\frac{2}{\pi} \int_{0}^{\theta_{c}} \mathrm{D}_{a}^{2} \mathrm{~d} \theta, \\
& \left\langle\hat{\rho}^{2}\right\rangle_{3 \mathrm{D}}=\left\langle\hat{\rho}_{e}^{2}\right\rangle_{3 \mathrm{D}}+\left\langle\hat{\rho}_{a}^{2}\right\rangle_{3 \mathrm{D}}=\int_{0}^{\pi / 2} \mathrm{D}_{e}^{2} \sin \theta \mathrm{d} \theta+\int_{0}^{\theta_{c}} \mathrm{D}_{a}^{2} \sin \theta \mathrm{d} \theta,
\end{aligned}
$$

where the far-field acoustic contribution satisfies $\mathbb{D}_{a}^{2}=\mathbb{U}_{a}^{2}+\mathbb{V}_{a}^{2}=\mathbb{P}_{a}^{2}$, and therefore

$$
\begin{aligned}
& \left\langle\hat{\rho}_{a}^{2}\right\rangle_{2 \mathrm{D}}=\left\langle\hat{u}_{a}^{2}\right\rangle_{2 \mathrm{D}}+\left\langle\hat{v}_{a}^{2}\right\rangle_{2 \mathrm{D}}=\left\langle\hat{p}^{2}\right\rangle_{2 \mathrm{D}}=\frac{2}{\pi} \int_{0}^{\theta_{c}} \mathrm{P}_{h}^{2} \mathrm{~d} \theta \\
& \left\langle\hat{\rho}_{a}^{2}\right\rangle_{3 \mathrm{D}}=\left\langle\hat{u}_{a}^{2}\right\rangle_{3 \mathrm{D}}+\left\langle\hat{v}_{a}^{2}\right\rangle_{3 \mathrm{D}}=\left\langle\hat{p}^{2}\right\rangle_{3 \mathrm{D}}=\int_{0}^{\theta_{c}} \mathbb{P}_{h}^{2} \sin \theta \mathrm{d} \theta .
\end{aligned}
$$

The averaged vorticity generated by the detonation front is computed in Fig. 5 (ab) in both two-dimensional (a) and three-dimensional (c) configurations as a function of the overdrive parameter $\mathcal{M}_{u} / \mathcal{M}_{\mathrm{cj}}-1$. The amplification of the entropic density 
fluctuations is shown in Fig. 5 (c-d). As noted for the turbulent kinetic energy, the highest level of vorticity generation is given when heat release perturbations upstream are negligible, i.e., when the mixture is in the heat-release peak. This case, which is in black lines, is similar to that found in Huete et al. (2013). The opposite limit, which occurs for same-density gases in sufficiently rich or lean conditions, shows a peak in the enstrophy generation at finite degrees of overdrive $\mathcal{M}_{u} / \mathcal{M}_{\mathrm{cj}}-1 \sim 1$ (see the green curve with its own log-log scale). Expectedly, when heat release variations are comparable to density perturbations, negative correlations provide higher amplitudes for the vorticity perturbations downstream. It resembles the findings in Sinha (2012), where the baroclinic torque due to a mean pressure gradient has an appreciable effect for non-zero entropic fluctuations in the upstream flow. As shown in Fig. 3, the highly overdriven limit for $|W| \gg|H|$ doubles the value for $|W|=|H|$.

Regarding the change in the entropic heterogeneities due to the detonation passage (in terms of the dimensionless variables measured with the bulk density), it is observed that overdrive modifies the hierarchy of the dominant contributions in the generation of entropy. For weak overdrives, $\mathcal{M}_{u} / \mathcal{M}_{\mathrm{cj}}-1 \ll 1$, negative correlations render the highest levels of entropy, while positive correlations yield the lowest. For $\mathcal{M}_{u} / \mathcal{M}_{\mathrm{cj}} \gg 1$, these contributions merge to the same asymptotic value, yielding half of that for $|W| \gg|H|$. The highest and lowest levels of entropy are obtained for $|W| \gg|H|$ and $|H| \gg|W|$, respectively, in the highly overdriven limit.

The results shown in Figs. 3 [5 are qualitatively similar to those obtained previously for the interaction of detonations with random vorticity perturbations (Jackson et al. 1993. Massa et al. 2011; Massa \& Lu 2011, Huete et al. 2017). However, a detailed comparison requires taking into account the diverse criteria used for normalization in different investigations. For isotropic vorticity fields, the upstream perturbed velocity field was scaled in Jackson et al. (1993); Huete et al. (2017) with the speed of sound downstream from the detonation, which always increases with the detonation intensity. If it had been scaled with the upstream sound speed, the results would have shown how the downstream perturbations decay with the overdrive to become negligibly small for strongly overdriven detonations. In strong-shock conditions, upstream perturbations in density and heat-release will dominate the turbulence generation across the detonation wave. In this particular case, the turbulence amplification factors measure how much turbulence is generated for any convective perturbation of the fuel mass fraction of order $\epsilon / \sqrt{W^{2}+H^{2}}$. When $W$ and/or $H$ are large, the allowed fuel mass fraction perturbations must be much smaller than the corresponding density and/or heat-release perturbations. This condition relaxes when $W$ and $H$ are of order unity or less. According to Table 1 , the value of $W$ is of order unity for light hydrocarbons and reasonably small for acetylene, while the value of $H$ is very sensitive to mixture conditions, being of order unity or less only in the vicinity of the heat release peak.

\subsection{Turbulence scales characterization}

Previous results refer to far-field mean values, which are independent of the shape of energy spectrum, on condition that it is isotropic. The analysis is valid at a distance sufficiently far from the detonation wave, where the vanishing contribution of the acoustic energy can be neglected. Close to the detonation wave, however, the decaying pressure contribution should be taken into account as described in A18 in the appendix for monochromatic perturbations.

Near-field contributions are studied in detailed in Sinha (2012); Quadros et al. $(2016)$; Sethuraman et al. (2018) for inert-shocks in canonical turbulent flows. To illustrate this effect, the emphasis is placed here on the near-field acoustic energy, which can 
weakly overdriven

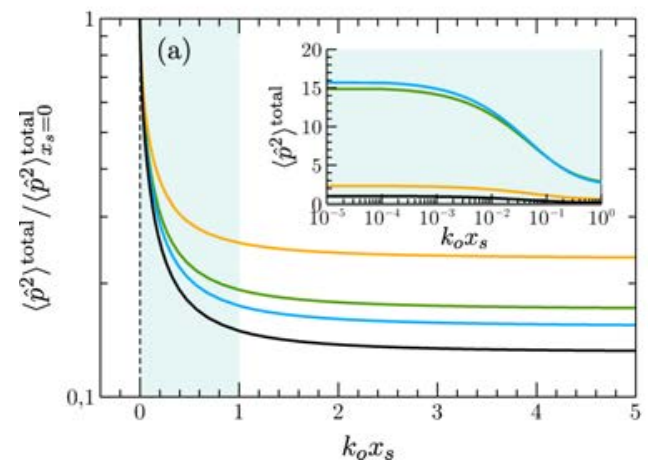

strongly overdriven

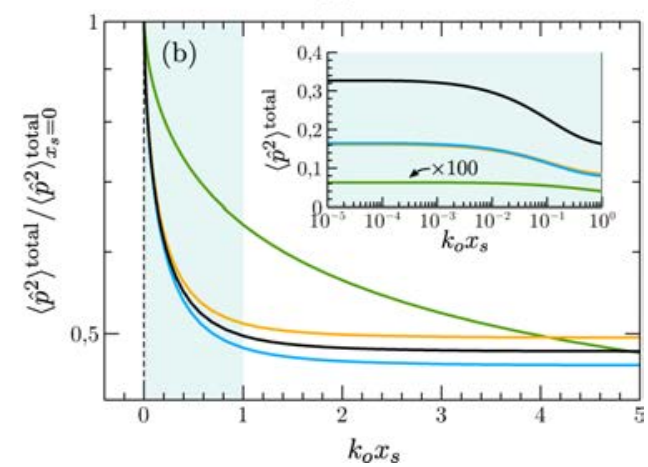

Figure 6: Three-dimensional total acoustic energy $\left\langle\hat{p}^{2}\right\rangle^{\text {total }} /\left\langle\hat{p}^{2}\right\rangle_{x_{s}=0}^{\text {total }}$ as a function of the scaled distance $k_{o} x_{s}$. Computations are given for $\gamma=1.2, Q=1$, weakly overdriven detonations $\mathcal{M}_{u}=1.001 \mathcal{M}_{\mathrm{cj}}(\mathrm{a})$, and strongly overdriven detonations $\mathcal{M}_{u}=5 \mathcal{M}_{\mathrm{cj}}$ (b). The distinguished cases include $|W| \gg|H|(-),|H| \gg|W|(-), W=H(-)$ and $W=-H(-)$. The function $\left\langle\hat{p}^{2}\right\rangle^{\text {total }}$ is shown in the insets for the same conditions.

be expressed as the sum of the non-vanishing and the vanishing contributions, namely $\left\langle\hat{p}^{2}\right\rangle^{\text {total }}\left(x_{s}\right)=\left\langle\hat{p}^{2}\right\rangle^{\text {non-van }}+\left\langle\hat{p}^{2}\right\rangle^{\text {van }}\left(x_{s}\right)$, where $x_{s}=u_{d} t-x$ measures the distance from the detonation pointing backwards. The former, which is associated with high-frequency perturbations, is explicitly given in $(3.9)$, while the decaying contribution is given by low-frequency disturbances in the following form:

$$
\left\langle\hat{p}^{2}\right\rangle^{\operatorname{van}}\left(x_{s}\right)=\frac{\int_{0}^{\infty}\left[\int_{\theta_{c}}^{\pi / 2}\left(\mathbb{P}_{l 1}^{2}+\mathbb{P}_{l 2}^{2}\right) e^{-2 \sigma(\theta) m k_{o} x_{s}} \sin \theta \mathrm{d} \theta\right] \epsilon(m)^{2} m^{2} \mathrm{~d} m}{\int_{0}^{\infty} \epsilon(m)^{2} m^{2} \mathrm{~d} m}
$$

for a three dimensional isotropic spectrum $\epsilon(m)$ that is written in terms of the reduced wavenumber amplitude $m=k / k_{o}$. The function $\sigma(\theta)$, given in A 19, defines the spatial decay rate along the dimensionless spatial coordinate $k_{o} x_{s}$. The decay rate depends on the form of the energy spectrum, which ultimately shapes the contribution of the different mode angles $\theta$.

The reduced acoustic energy $\left\langle\hat{p}^{2}\right\rangle^{\text {total }} /\left\langle\hat{p}^{2}\right\rangle_{x_{s}=0}^{\text {total }}$ is shown in Fig. 6] as a function of the scaled distance $k_{o} x_{s}$. The functions are computed for $|W| \gg|H|$ (black lines), $|H| \gg|W|$ (green lines), $W=H$ (orange lines) and $W=-H$ (blue lines). Weakly overdriven detonations are displayed in panel (a) for $\mathcal{M}_{u}=1.001 \mathcal{M}_{\mathrm{cj}}$ and strongly overdriven detonations are shown in panel (b) for $\mathcal{M}_{u}=5 \mathcal{M}_{\text {cj. }}$. Except for the case $|H| \gg|W|$ in Fig. 6(b), which has been amplified by a factor of 100 in the inset, the decay rate is not significantly affected by the type of perturbation upstream. It takes a distance $x_{s} \sim k_{o}^{-1}$ for the acoustic energy to reach the asymptotic plateau corresponding to the far-field contribution. For weakly overdriven detonations, negative correlations exhibit the highest value of acoustic energy in the near-field, in contrast to the far-field solution where perturbations of the type $|H| \gg|W|$ render slightly higher values. On the other hand, upstream flows dominated by density perturbations $|W| \gg|H|$ produce the highest acoustic energy in the whole spatial domain. In general, the vanishing contribution $\left\langle\hat{p}^{2}\right\rangle$ van has a relatively low influence on the total pressure field for $k_{o} x_{s} \gtrsim 1$. Notice that near-field profiles are computed for purely inviscid flows. Depending on the particular conditions, there may exist another contribution coming from viscous dissipation that may not be neglected, as noted in Quadros et al. (2016); Sethuraman et al. (2018). 

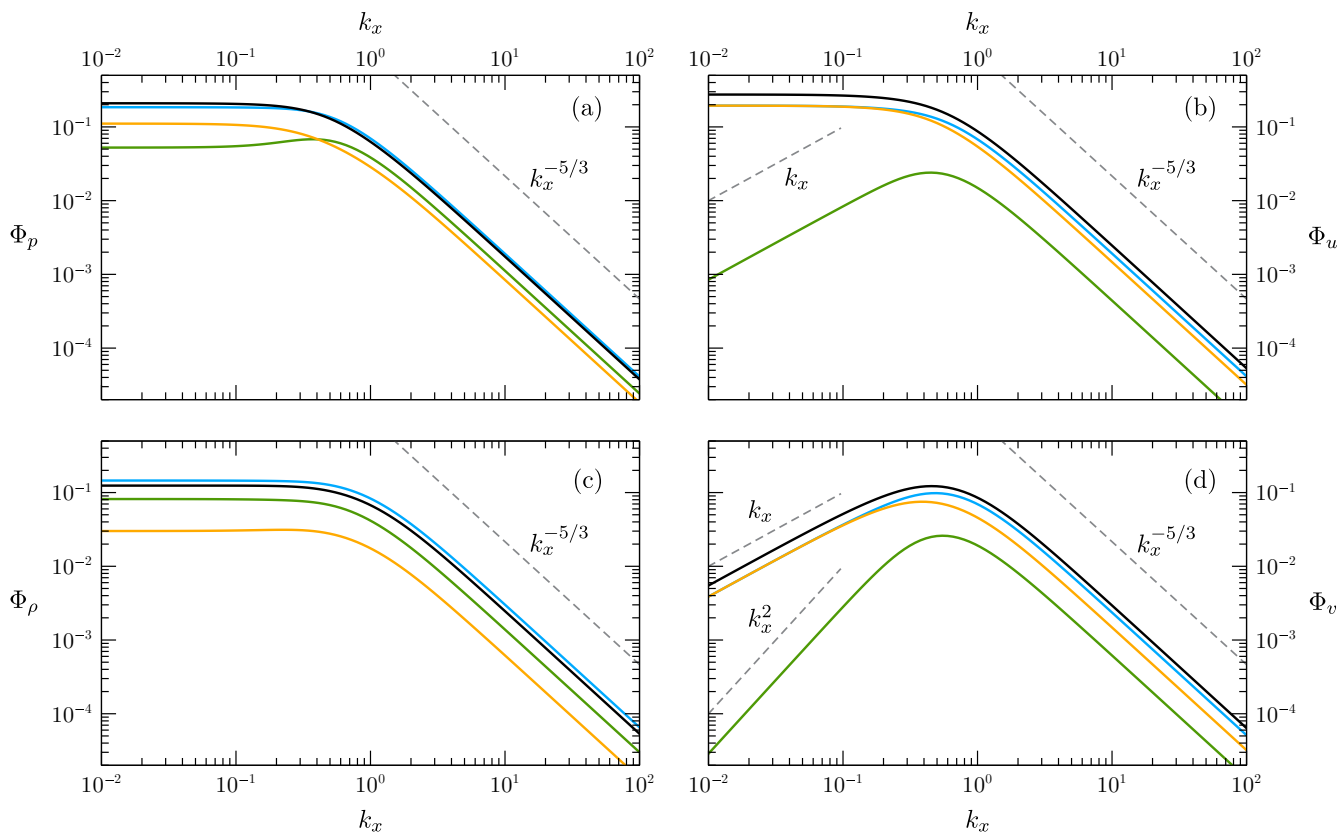

Figure 7: One-dimensional power spectra of the pressure (a), density (c) and longitudinal and transverse velocity components ( $b$ and $d$, respectively). Computations are shown as a function of the wavenumber $k_{x}$ for $\gamma=1.2, \mathcal{M}_{u}=1.2 \mathcal{M}_{\mathrm{cj}}, Q=1$, and $|W| \gg|H|$ $(-),|H| \gg|W|(-), W=H(-)$ and $W=-H(-)$.

To gain further insight into the properties of the turbulent flow that emerges far downstream the detonation wave, the one-dimensional power spectra of the different variables can be evaluated through the integral functions

$$
\begin{aligned}
& \Phi_{p}\left(k_{x}\right)=N k_{x}^{-5 / 3} \int_{0}^{\pi / 2} \mathbb{P}^{2}(\theta) \frac{\sin ^{3} \theta}{\cos ^{5} \theta\left(k_{x}^{-2}+1+\tan ^{2} \theta\right)^{17 / 6}} \mathrm{~d} \theta, \\
& \Phi_{u}\left(k_{x}\right)=N k_{x}^{-5 / 3} \int_{0}^{\pi / 2} \mathbb{U}^{2}(\theta) \frac{\sin ^{3} \theta}{\cos ^{5} \theta\left(k_{x}^{-2}+1+\tan ^{2} \theta\right)^{17 / 6}} \mathrm{~d} \theta, \\
& \Phi_{v}\left(k_{x}\right)=N k_{x}^{-5 / 3} \int_{0}^{\pi / 2} \mathbb{V}^{2}(\theta) \frac{\sin ^{3} \theta}{\cos ^{5} \theta\left(k_{x}^{-2}+1+\tan ^{2} \theta\right)^{17 / 6}} \mathrm{~d} \theta, \\
& \Phi_{\rho}\left(k_{x}\right)=N k_{x}^{-5 / 3} \int_{0}^{\pi / 2} \mathbb{D}^{2}(\theta) \frac{\sin ^{3} \theta}{\cos ^{5} \theta\left(k_{x}^{-2}+1+\tan ^{2} \theta\right)^{17 / 6}} \mathrm{~d} \theta,
\end{aligned}
$$

provided that the three-dimensional von Kármán model is applicable, as in Ribner (1987); Jackson et al. (1993), which translates into considering turbulence spectra of the form $\epsilon\left(k_{x}, k_{y}\right)^{2} \sim k_{y}^{2} /\left(1+k_{x}^{2}+k_{y}^{2}\right)^{17 / 6}$. The normalization parameter is chosen to satisfy

$$
N^{-1}=\int_{-\infty}^{\infty} \int_{0}^{\infty} \frac{k_{y}^{2}}{\left(1+k_{x}^{2}+k_{y}^{2}\right)^{17 / 6}} k_{y} \mathrm{~d} k_{y} \mathrm{~d} k_{x} .
$$

Previous investigations on the interaction of turbulence with adiabatic shocks have reported that large-scale turbulence is amplified to a greater extent than small-scale 
motions (Andreopoulos et al. 2000). A similar behaviour is observed with detonations when they travel in isotropic vorticity flows (Jackson et al. 1993$)$ or isotropic entropicdensity flows, as the example considered here for $|W| \gg|H|$. This phenomenon is readily observed in the black curves plotted in Fig. 7, where the power spectra $\Phi_{p}, \Phi_{u}, \Phi_{v}$ and $\Phi_{\rho}$ are represented as a function of the longitudinal wavenumber $k_{x}$ for $\gamma=1.2$, $\mathcal{M}_{u}=1.2 \mathcal{M}_{\mathrm{cj}}, Q=1$, and for $|W| \gg|H|$ (black lines), $|H| \gg|W|$ (green lines), $W=H$ (orange lines) and $W=-H$ (blue lines). In the presence of upstream density perturbations $W$ with values of $H$ that are either negligible or of the same order, the curves exhibit a similar pattern, and the case $|W| \gg|H|$ results in the highest values along with the negatively correlated cases $W=-H$. As a result of the destructive interference, positive correlations yield lower values in most of the domains for each spectrum. The case $|H| \gg|W|$ results in the lowest intensity for the two velocity spectra in all the domains and for the pressure spectrum for small wave numbers only. Regarding this large-scale limit, it is noticeable that curves of $\Phi_{u}$ approach a plateau as $k_{x}$ decreases except for the case $|H| \gg|W|$, where the spectrum decreases as $k_{x}^{-1}$. Curves for $\Phi_{v}$ decrease as $k_{x}$ is reduced in all cases ; however, the isochoric case shows a higher slope $k_{x}^{-2}$. Regarding $\Phi_{p}$ and $\Phi_{\rho}$, they reach plateaus in the large-scale limit for any combination of $W$ and $H$. With respect to the small-scale regime, all contributions obey the von

Kármán decay law $k_{x}^{-5 / 3}$. Although these conclusions apply for $\mathcal{M}_{u}=1.2 \mathcal{M}_{\text {cj }}$, they can be extrapolated to higher overdrives. On the other hand, the weakly overdriven limit should be discussed separately, as the acoustic part may change the ranking of the most important contributions.

Aside from the fact that viscosity and molecular diffusion are not accounted for in the model - in which case the spectra would become time-dependent functions after the passage of the detonation (Sinha 2012, Sethuraman \& Sinha 2020) - , the small-scale regime must be taken with caution as the thin-detonation hypothesis may not be fulfilled. Therefore, the properties of the turbulent flow behind the detonation front predicted by the linear interaction theory should be further extended to include the effects of finite reaction lengths, as noted in Massa et al. (2011); Massa \& Lu (2011), and non-linear contributions, as noted in Larsson \& Lele (2009); Prakash \& Raman (2019); Livescu (2020); Tian et al. (2020). The latter is unavoidable if multi-phase environments are under consideration (Watanabe et al. 2019,2020 ). However, there are some aspects predicted by the model that deserve particular attention. When a detonation wave or a reactive shock is considered, the possibility of having different heat releases across the detonation wave due to non-perfect mixing should not be neglected. This effect plays a leading role in the turbulence generation process of acoustic type in the CJ condition. As a result, the turbulent flow downstream cannot be assumed to be isobaric, as reasonably done for inert shocks. For detonations, the dilatational part of the velocity gradient tensor cannot be neglected when the correlations between the corresponding invariants are employed to characterize the topology of the turbulent flow.

\section{Corrections to Rankine-Hugoniot equations and propagation speed}

The analysis presented above demonstrates how a non-uniform fresh mixture that exhibits weak fuel mass fraction deviations perturbs the detonation front surface, thereby creating additional disturbances in the burnt gas. The deposition of rotational/acoustic velocity perturbations in the downstream flow along with the amplification of the entropic fluctuations comes with a price in the overall $\mathrm{RH}$ relations. In particular, changes are expected in the jump conditions given the averaged downstream magnitudes with respect to the case of uniform mixtures with the same averaged upstream properties (Lele 1992). 
As a direct consequence, the averaged propagation speed of the detonation wave will also be affected, as previously noted for inert shocks (Hazak et al. 1998; Velikovich et al. 2012).

The goal of this section is to predict these changes and to provide analytical expressions, which are similar to those reported in Velikovich et al. (2012) in terms of the parameters characterizing the detonation front. Then, the non-uniformity parameter

$$
\bar{\epsilon}=\sqrt{1-\frac{\left\langle Y_{u}\right\rangle^{2}}{\left\langle Y_{u}^{2}\right\rangle}}
$$

is introduced to characterize the average fuel mass fraction fluctuations. Although it may vary from 0 to 1 , the upper limit corresponding to infinite density gradients as those present in multi-phase flow, the use of the linear model previously presented forces it to be much smaller than unity and therefore equal to $\sqrt{\left\langle\delta Y_{u}^{2}\right\rangle}$ in 2.2 .

\subsection{Averaged turbulent Rankine-Hugoniot jump equations}

The changes in the $\mathrm{RH}$ relations are given by second-order corrections in the form

$$
\frac{\left\langle\rho_{d}\right\rangle}{\left\langle\rho_{u}\right\rangle}=\mathcal{R}_{d}\left(1+\bar{\epsilon}^{2} \delta \mathcal{R}\right), \quad \frac{\left\langle p_{d}\right\rangle}{p_{u}}=\mathcal{P}_{d}\left(1+\bar{\epsilon}^{2} \delta \mathcal{P}\right), \quad \frac{\left\langle u_{d}\right\rangle}{u_{u}}=\frac{1}{\mathcal{R}_{d}}\left(1+\bar{\epsilon}^{2} \delta \mathcal{U}\right),
$$

provided that the flow perturbations can be written as the breakdown of averaged and local deviation contributions, e.g., $\rho_{d}=\left\langle\rho_{d}\right\rangle(1+\bar{\epsilon} \hat{\rho})$ for the burnt-gas density field. To illustrate this, Fig. 8 (left) sketches the effect of the second-order corrections to the postdetonation $\mathrm{RH}$ values. Hollow circles represent the initial conditions, which may also be different if second-order average values are non-zero. The blue and orange filled circles indicate the one-dimensional and turbulent post-detonation conditions, respectively. The vector $(\delta \mathcal{V}, \delta \mathcal{P})$, with $\delta \mathcal{V}=-\delta \mathcal{R}$ standing for the correction to the specific volume, is defined to characterize the relative deviations of the post-detonation $\mathrm{RH}$ state on the $\left(V / V_{u}, p / p_{u}\right)$-plane. The right panel shows the vector $(\delta \mathcal{V}, \delta \mathcal{P})$ as computed from the theory presented below for the same conditions given in Fig. 9, namely, $\gamma=1.2$, $\mathcal{M}_{u}=1.2 \mathcal{M}_{\mathrm{cj}}$ and $Q=1$. In this case, both pressure and density corrections are negative in the whole domain, yet the values differ significantly depending on the particular values of $W$ and $H$. Note also that in agreement with the results of Section 3, perturbations weaken for $|H| \gg W$ and intensify for $|H| \ll W$.

Corrections to the $\mathrm{RH}$ jump conditions due to weak turbulence are derived from the lowest non-vanishing order, i.e., the non-linear second-order terms describing the interaction between the different perturbation modes and their self-interactions. The natures of rotational and entropic modes are similar as they are both carried along with the fluid. Thus, they correlate and provide rotational-entropic and rotationalrotational second-order corrections. By contrast, acoustic waves are uncorrelated with the entropic and rotational perturbations. As a consequence, second-order corrections can be decomposed into entropic-rotational and acoustic-acoustic terms, separately.

The entropic-rotational correlations enter only as second-order corrections as the first order of the random-phase oscillating terms vanishes. Conversely, acoustic perturbations play a two-fold role in the global contribution. The first role, which is akin to entropicrotational modes, is given by second-order corrections to the mass, momentum and energy fluxes across the detonation front. The second role stems from the fact that acoustic waves shift the average values of the flow variables in the second-order of the sound amplitude. Further details of the analytical derivation of the distinguished contributions can be withdrawn from Velikovich et al. (2012) and references therein, particularly the 

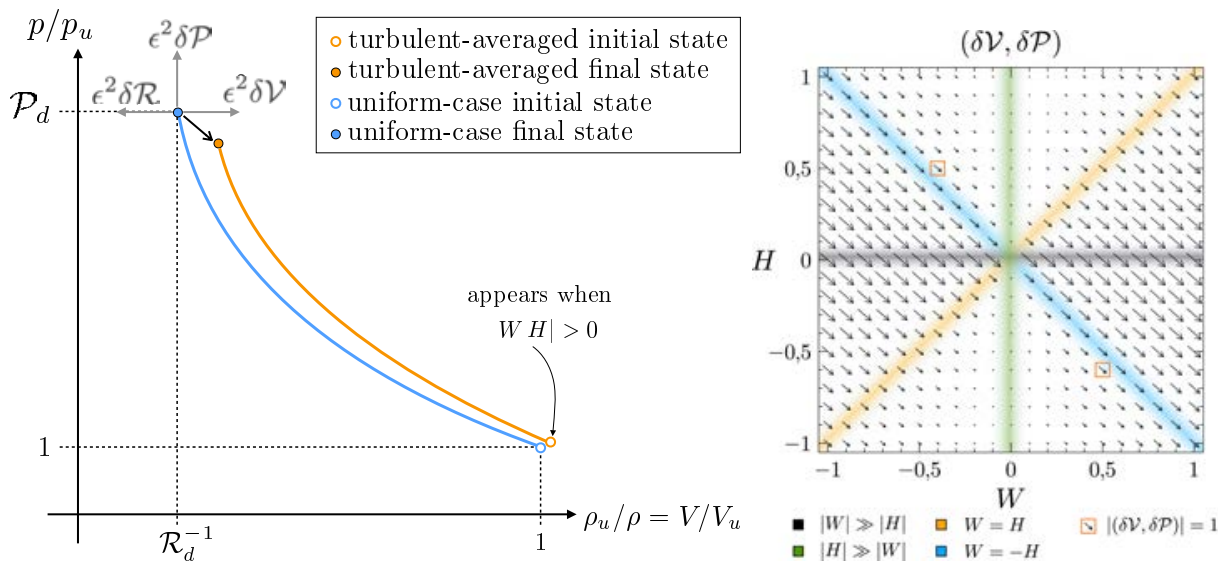

Figure 8: Left: Sketch for the Rankine-Hugoniot curves. In blue the one-dimensional curve and in orange the second-order-averaged curve. Right: Computations for $\gamma=1.2$, $\mathcal{M}_{u}=1.2 \mathcal{M}_{\mathrm{cj}}, Q=1, H=[-1: 1]$ and $W=[-1: 1]$.

supplemental material, as well as in Westervelt (1950); Blackstock (1962); Ostrovskii (1968).

As a result of the second-order corrections to the mass, momentum and energy fluxes across the detonation wave, the downstream pressure, density and streamwise velocity suffer the corresponding corrections $\delta \mathcal{P}_{\mathrm{f}}, \delta \mathcal{R}_{\mathrm{f}}$ and $\delta \mathcal{U}_{\mathrm{f}}$, where the subscript $\mathrm{f}$ is used to denote changes due to flux corrections. These corrections can be obtained from the averaged turbulent conservation equations, namely

$$
\begin{aligned}
\left\langle\rho_{u} u_{u}\right\rangle & =\left\langle\rho_{d} u_{d}\right\rangle \\
\left\langle p_{u}+\rho_{u} u_{u}^{2}\right\rangle & =\left\langle p_{d}+\rho_{d} u_{d}^{2}\right\rangle \\
\left\langle\frac{\gamma}{\gamma-1} p_{u} u_{u}+\frac{1}{2} \rho_{u} u_{u}^{3}+\rho_{u} u_{u} q_{u}\right\rangle & =\left\langle\frac{\gamma}{\gamma-1} p_{d} u_{d}+\frac{1}{2} \rho_{d} u_{d}\left(u_{d}^{2}+v_{d}^{2}\right)\right\rangle,
\end{aligned}
$$

which provide, with use made of (4.2) and knowing that first-order entropic and rotational corrections give null average contributions, the following second-order relationships:

$$
\begin{aligned}
\delta \mathcal{R}_{\mathrm{f}}+\delta \mathcal{U}_{\mathrm{f}}+\mathcal{M}_{d}^{-1}\left\langle l_{\mathrm{f}}\right\rangle & =0 \\
\delta \mathcal{P}_{\mathrm{f}}+\gamma \mathcal{M}_{d}^{2}\left(\delta \mathcal{R}_{\mathrm{f}}+2 \delta \mathcal{U}_{\mathrm{f}}\right)+\gamma\left\langle m_{\mathrm{f}}\right\rangle & =0 \\
\mathcal{M}_{d}^{2} \delta \mathcal{R}_{\mathrm{f}}+\left(3 \mathcal{M}_{d}^{2}+\frac{2}{\gamma-1}\right) \delta \mathcal{U}_{\mathrm{f}}+\frac{2}{\gamma-1} \delta \mathcal{P}_{\mathrm{f}}+\frac{2}{(\gamma-1) \mathcal{M}_{d}}\left\langle n_{\mathrm{f}}\right\rangle & =q_{\mathrm{wh}},
\end{aligned}
$$

where the factor

$$
q_{\mathrm{wh}}=\frac{4 Q \mathcal{M}_{d}^{2} \mathcal{R}_{d}^{2}}{\left(\gamma^{2}-1\right) \mathcal{M}_{u}^{2}}\left\langle\hat{\rho}_{u} \hat{q}_{u}\right\rangle=\frac{4 Q \mathcal{R}_{d}}{\left(\gamma^{2}-1\right) \mathcal{P}_{d}} \frac{W H}{W^{2}+H^{2}}
$$

appears due to the existence of two non-vanishing second-order sources of perturbations upstream. The factor $q_{\mathrm{wh}}$ vanishes for flows with uniform density or constant heat-release given that first-order averages of entropic disturbances do not provide net contributions. The terms $\left\langle l_{\mathrm{f}}\right\rangle,\left\langle m_{\mathrm{f}}\right\rangle$, and $\left\langle n_{\mathrm{f}}\right\rangle$ represent the dimensionless second-order turbulent corrections to the mass, streamwise momentum, and energy fluxes, respectively. As mentioned above, the terms split into entropic-rotational (subscript er) and acoustic- 
acoustic (subscript $a a$ ) contributions to yield the following:

$$
\begin{aligned}
\left\langle l_{\mathrm{f}}\right\rangle= & \left\langle l_{e r}\right\rangle+\left\langle l_{a a}\right\rangle=\left\langle\hat{\rho}_{e} \hat{u}_{r}\right\rangle-\frac{\gamma+1}{4} \mathcal{M}_{d}\left\langle\hat{p}^{2}\right\rangle \\
\left\langle m_{\mathrm{f}}\right\rangle= & \left\langle m_{e r}\right\rangle+\left\langle m_{a a}\right\rangle=2 \mathcal{M}_{d}\left\langle\hat{\rho}_{e} \hat{u}_{r}\right\rangle+\left\langle\hat{u}_{r}^{2}\right\rangle-\frac{(\gamma-1) \mathcal{M}_{d}^{2}+3-\gamma}{4}\left\langle\hat{p}^{2}\right\rangle+\left\langle\hat{u}_{a}^{2}\right\rangle, \\
\left\langle n_{\mathrm{f}}\right\rangle= & \left\langle n_{e r}\right\rangle+\left\langle n_{a a}\right\rangle=\frac{\mathcal{M}_{d}(\gamma-1)}{2}\left[3\left\langle\hat{u}_{r}^{2}\right\rangle+\left\langle\hat{v}_{r}^{2}\right\rangle+3 \mathcal{M}_{d}\left\langle\tilde{\rho}_{e} \tilde{u}_{r}\right\rangle\right]- \\
& -\frac{(\gamma+1) \mathcal{M}_{d}}{8}\left[(\gamma-1) \mathcal{M}_{d}^{2}+2(2-\gamma)\right]\left\langle\hat{p}^{2}\right\rangle+(\gamma-1) \mathcal{M}_{d}\left\langle\hat{u}_{a}^{2}\right\rangle-(\gamma-1)\left\langle\hat{p} \hat{u}_{a}\right\rangle,
\end{aligned}
$$

where $\left\langle\hat{u}_{r}^{2}\right\rangle,\left\langle\hat{u}_{a}^{2}\right\rangle,\left\langle\hat{v}_{r}^{2}\right\rangle$, and $\left\langle\hat{p}^{2}\right\rangle$ have been previously defined in 3.5], 3.6) and 3.9. The averages $\left\langle\tilde{\rho}_{e} \tilde{u}_{r}\right\rangle$ and $\left\langle\hat{p} \hat{u}_{a}\right\rangle=\left\langle\hat{\rho}_{a} \hat{u}_{a}\right\rangle$ are second-order contributions that have not been previously introduced. As noted in (3.5), (3.6) and (3.9), these parameters can be written in terms of integral expressions for two-dimensional and three-dimensional upstream isotropic spectra, namely

$$
\begin{aligned}
& \left\langle\hat{\rho}_{e} \hat{u}_{r}\right\rangle_{2 \mathrm{D}}=-\frac{2}{\pi} \int_{0}^{\theta_{c}} \mathbb{D}_{e, h} \mathbb{U}_{r, h} \mathrm{~d} \theta-\frac{2}{\pi} \int_{\theta_{c}}^{\pi / 2}\left[\mathbb{D}_{e, l 1} \mathbb{U}_{r, l 1}+\mathbb{D}_{e, l 2} \mathbb{U}_{r, l 2}\right] \mathrm{d} \theta, \\
& \left\langle\hat{\rho}_{e} \hat{u}_{r}\right\rangle_{3 \mathrm{D}}=-\int_{0}^{\theta_{c}} \mathbb{D}_{e, h} \mathbb{U}_{r, h} \sin \theta \mathrm{d} \theta-\int_{\theta_{c}}^{\pi / 2}\left[\mathbb{D}_{e, l 1} \mathbb{U}_{r, l 1}+\mathbb{D}_{e, l 2} \mathbb{U}_{r, l 2}\right] \sin \theta \mathrm{d} \theta,
\end{aligned}
$$

and

$$
\left\langle\hat{p} \hat{u}_{a}\right\rangle_{2 \mathrm{D}}=\frac{2}{\pi} \int_{0}^{\theta_{c}} \mathbb{P}_{h} \mathbb{U}_{a} \mathrm{~d} \theta, \quad\left\langle\hat{p} \hat{u}_{a}\right\rangle_{3 \mathrm{D}}=\int_{0}^{\theta_{c}} \mathbb{P}_{h} \mathbb{U}_{a} \sin \theta \mathrm{d} \theta
$$

for the entropic-rotational contribution to the mass flux and the projected acoustic contribution to the energy flux, respectively. The amplitudes of the entropic density and the rotational streamwise velocity are given in $\mathrm{A} 22$ and $\mathrm{A} 29$, respectively, and the negative sign arises from the opposite direction between the flow and the orientation of the detonation front. Finally, the amplitude of the pressure perturbations $\mathbb{P}_{h}$ together with $\mathbb{U}_{a}=\mathbb{P}_{h} \kappa_{a} / \omega_{a}$ is given in $\left(\mathrm{A} 9\right.$. It is readily observed that $\left\langle\hat{\rho}_{e} \hat{u}_{r}\right\rangle$ is directly associated to the far-field convective energy flux, which is studied in detailed in Quadros et al. (2016); Sethuraman et al. (2018) for different types of spectra in canonical shockturbulence scenarios.

The post-shock density, pressure, and streamwise velocity component exhibit secondorder changes due to the presence of the acoustic wave field. The dual temporal-spatial averaging that is conveniently applied to this problem is similar to time averaging performed in the reference frame with the gas at rest. The averaging must be computed in the Eulerian reference system at a fixed position relative to the detonation front. Therefore, as a direct consequence of the shift in the acoustic waves, there exists an additional contribution in the burnt-gas properties, namely

$$
\delta \mathcal{R}_{\mathrm{b}}=-\frac{\gamma+1}{4}\left\langle\hat{p}^{2}\right\rangle, \quad \delta \mathcal{P}_{\mathrm{b}}=-\frac{\gamma(3-\gamma)}{4}\left\langle\hat{p}^{2}\right\rangle, \quad \delta \mathcal{U}_{\mathrm{b}}=\frac{1}{\mathcal{M}_{d}}\left\langle\hat{p} \hat{u}_{a}\right\rangle,
$$

where the subscript b denotes changes in the background downstream properties due to random-phase acoustic perturbations. The ultimate corrections to the $\mathrm{RH}$ jump conditions are given by the sum of the contributions due to the turbulent fluxes (subscript f) and background corrections induced by the acoustic field (subscript b). With use made 

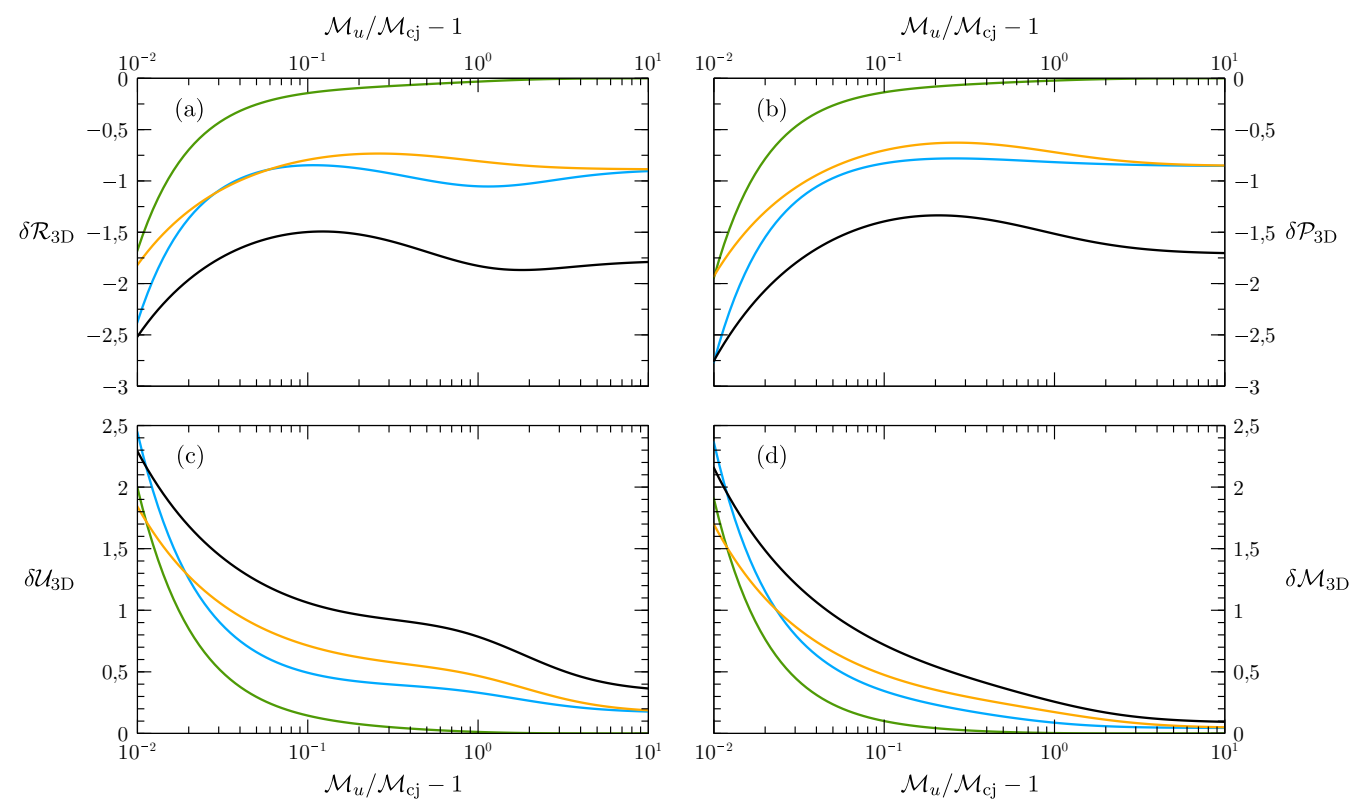

Figure 9: Three-dimensional second-order correction to the RH jump conditions (a) $\delta \mathcal{R}$, (b) $\delta \mathcal{U}$, (c) $\delta \mathcal{P}$, and (d) $\delta \mathcal{M}$ as a function of the overdrive parameter $\mathcal{M}_{u} / \mathcal{M}_{\mathrm{cj}}-1$. Computations correspond to $\gamma=1.2, Q=1$, and $|W| \gg|H|(-),|H| \gg|W|(-)$, $W=H(-)$ and $W=-H(-)$.

of 4.4, 4.6 and 4.9, the turbulent corrections to the $\mathrm{RH}$ conditions read as follows:

$$
\begin{gathered}
\delta \mathcal{R}=\delta \mathcal{R}_{\mathrm{f}}+\delta \mathcal{R}_{\mathrm{b}}=\frac{1}{1-\mathcal{M}_{d}^{2}}\left[\frac{\mathcal{M}_{d}^{2}(\gamma+3)-2}{2 \mathcal{M}_{d}}\left\langle l_{\mathrm{f}}\right\rangle-\gamma\left\langle m_{\mathrm{f}}\right\rangle+\frac{1}{\mathcal{M}_{d}}\left\langle n_{\mathrm{f}}\right\rangle-\frac{\gamma-1}{2} q_{\mathrm{wh}}\right] \\
\delta \mathcal{P}=\delta \mathcal{P}_{\mathrm{f}}+\delta \mathcal{P}_{\mathrm{b}}=\frac{\gamma}{1-\mathcal{M}_{d}^{2}}\left[\frac{\mathcal{M}_{d}\left[\mathcal{M}_{d}^{2}(\gamma-1)+2\right]}{2}\left\langle l_{\mathrm{f}}\right\rangle-\left[\mathcal{M}_{d}^{2}(\gamma-1)+1\right]\left\langle m_{\mathrm{f}}\right\rangle\right. \\
\left.+\mathcal{M}_{d}\left\langle n_{\mathrm{f}}\right\rangle-\frac{\mathcal{M}_{d}^{2}(\gamma-1)}{2} q_{\mathrm{wh}}\right]-\frac{\gamma(3-\gamma)}{4}\left\langle\hat{p}^{2}\right\rangle, \quad(4.11 \\
\delta \mathcal{U}=\delta \mathcal{U}_{\mathrm{f}}+\delta \mathcal{U}_{\mathrm{b}}=\frac{-1}{1-\mathcal{M}_{d}^{2}}\left[\frac{\mathcal{M}_{d}(\gamma+1)}{2}\left\langle l_{\mathrm{f}}\right\rangle-\gamma\left\langle m_{\mathrm{f}}\right\rangle+\frac{1}{\mathcal{M}_{d}}\left\langle n_{\mathrm{f}}\right\rangle-\frac{\gamma-1}{2} q_{\mathrm{wh}}\right] \\
+\frac{1}{\mathcal{M}_{d}}\left\langle\hat{p} \hat{u}_{a}\right\rangle . \quad(4.12
\end{gathered}
$$

Associated to the turbulence-induced changes in the RH jump conditions, the Mach number of the burnt gas fluid particles is also modified according to

$$
\delta \mathcal{M}=\frac{\mathcal{M}_{u}}{\sqrt{\mathcal{R}_{d} \mathcal{P}_{d}}}\left(\delta \mathcal{U}+\frac{1}{2} \delta \mathcal{R}-\frac{1}{2} \delta \mathcal{P}\right) .
$$

Computations of $\delta \mathcal{R}, \delta \mathcal{P}, \delta \mathcal{U}$ and $\delta \mathcal{M}$ are represented in Fig. 9 as a function of the overdrive parameter $\mathcal{M}_{u} / \mathcal{M}_{\mathrm{cj}}-1$ for three-dimensional isotropic perturbations with $\gamma=$ 
1.2 , and $Q=1$. The four distinguished cases include $|W| \gg|H|$ (black lines), $|H| \gg|W|$ (green lines), $W=H$ (orange lines) and $W=-H$ (blue lines). Density and pressure corrections, $\delta \mathcal{R}$ and $\delta \mathcal{P}$, are always negative, and the amplitude of the second-order corrections weakens with the overdrive and approaches an asymptotic constant value that is null only when $W=0$, which is consistent with previous turbulence generation functions. A qualitative representation of the combined effect of $\delta \mathcal{P}$ and $\delta \mathcal{R}$ is shown in Fig. 8 (right) in terms of the vector $(\delta \mathcal{V}, \delta \mathcal{P})$, with $\delta \mathcal{V}=-\delta \mathcal{R}$. Curves of the postdetonation velocity and corresponding Mach number go in the opposite direction because $\delta \mathcal{U}$ and $\delta \mathcal{M}$ exhibit positive values in all cases. For the conditions chosen in Fig. 9 , perturbations involving only density changes result in the strongest corrections, while those involving only variations in heat-release yield the weakest corrections far from the CJ condition. Positive and negative correlations, on the other hand, yield intermediate values for the second-order corrections.

\subsection{Detonation propagation speed}

The averaged turbulent properties of the post-detonation state differ from those of the unperturbed, i.e., one-dimensional case. Second-order corrections for density, pressure and velocity must be accounted for to properly describe the burnt-gas state. In real conditions, depending on the experimental setup, boundary conditions may impose different restrictions to the burnt-gas flow. For example, a highly overdriven detonation formed by a piston-driven shock traveling into the reactive mixture forces the velocity of the burnt gas particles to be equal to the piston velocity, $u_{p}=u_{u}-u_{d}$, in steady state. Then, along with the velocity variation of the expelled burnt gas $\delta \mathcal{U}$, a detonation propagation speed correction must occur to satisfy the boundary condition, i.e., $\delta\left\langle u_{p}\right\rangle=0=\delta u_{u}-\bar{\epsilon}^{2} u_{d} \delta \mathcal{U}$, which yields

$$
\left.\delta \mathcal{S}\right|_{\delta\left\langle u_{p}\right\rangle=0}=\left.\frac{1}{\bar{\epsilon}^{2}} \frac{\delta u_{u}}{u_{u}}\right|_{\delta\left\langle u_{p}\right\rangle=0}=\frac{1}{\mathcal{R}_{d}} \delta \mathcal{U},
$$

where $\delta \mathcal{S}$ is an order-of-unity function that characterize the correction of the detonation propagation speed. According to the results shown in Fig. 9, where $\delta \mathcal{U}$ is positive, the accommodated velocity of the detonation is therefore increased. Except for weakly overdriven detonations, where negative correlated perturbations $W=-H$ render the strongest correction, the case corresponding to dominant density perturbations $W \gg H$ is the case where the detonation would move faster compared to the homogeneous case.

Another canonical possibility is that total pressure (including corrections) must remain invariant. For this to be true, $\delta\left\langle p_{d}\right\rangle=0=p_{d} \bar{\epsilon}^{2} \delta \mathcal{P}+\left(\mathrm{d} p_{d}\right) /\left(\mathrm{d} u_{u}\right) \delta u_{u}$ and the detonation propagation speed must be adapted according to

$$
\left.\delta \mathcal{S}\right|_{\delta\left\langle p_{d}\right\rangle=0}=\left.\frac{1}{\bar{\epsilon}^{2}} \frac{\delta u_{u}}{u_{u}}\right|_{\delta\left\langle p_{d}\right\rangle=0}=-\frac{\mathcal{P}_{d}}{\mathcal{M}_{u}}\left(\frac{\mathrm{d} \mathcal{P}_{d}}{\mathrm{~d} \mathcal{M}_{u}}\right)^{-1} \delta \mathcal{P},
$$

where $\left(\mathrm{d} \mathcal{P}_{d}\right) /\left(\mathrm{d} \mathcal{M}_{u}\right)>0$ and $\delta \mathcal{P}<0$, according to Fig. 9. Then, the constant-pressure boundary condition also predicts a positive second-order correction to the propagation speed, which aligns with the previous boundary condition. These considerations oppose the initially imposed isolated-wave boundary condition. For 4.14 ) and (4.15) to be true along with the isolated condition, the influence of the coupling surface must be sufficiently far away from the detonation for the asymptotic oscillating regime to be achieved before the reflections come into play. Certainly, more complex scenarios may require non-canonical boundary conditions to be imposed. For example, for a detonation moving along a tube with the back extreme being open, the boundary condition should 

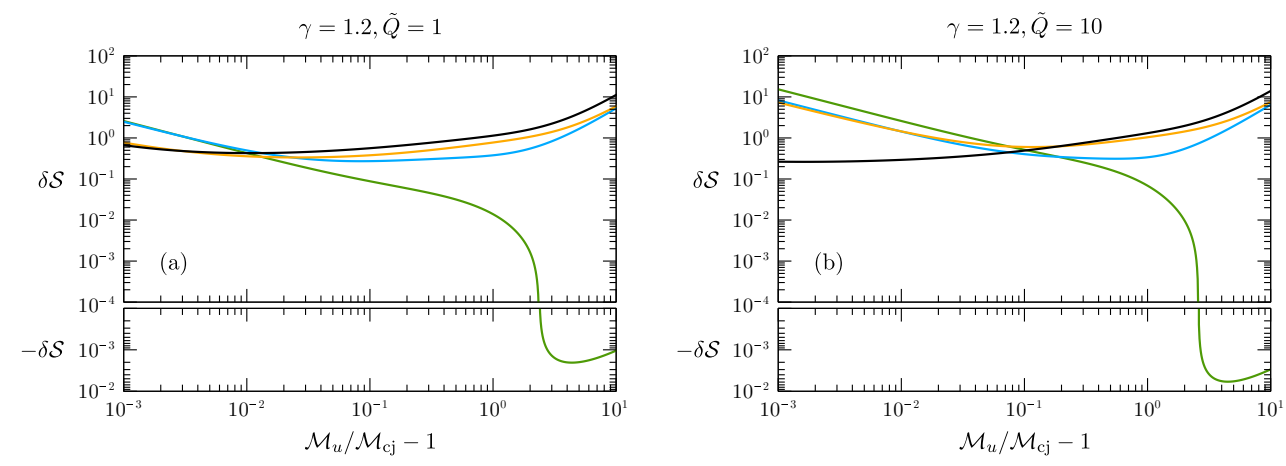

Figure 10: Three-dimensional second-order correction of the detonation propagation velocity $\delta \mathcal{S}$, according to 4.16 , as a function of the overdrive parameter $\mathcal{M}_{u} / \mathcal{M}_{\mathrm{cj}}-1$. Computations are provided for $\gamma=1.2, \tilde{Q}=1$ (a) and $\tilde{Q}=10$ (b) evaluated for $|W| \gg|H|(-),|H| \gg|W|(-), W=H(-)$ and $W=-H(-)$.

be given by the corresponding matching of the far-field non-reflective condition with the acoustics inside the channel because both velocity and pressure variations are non-zero $\delta\left\langle u_{p}\right\rangle \neq \delta\left\langle p_{d}\right\rangle \neq 0$ downstream.

Regarding detonations traveling close to the CJ velocity, $1-\mathcal{M}_{d} \ll 1$, the closure is given by imposing $\delta\left\langle\mathcal{M}_{d}\right\rangle=0$, i.e., not admitting variations in the post-detonation Mach number as the reacting gas expands up to its maximum value $\mathcal{M}_{d}=1$. Since the corresponding second-order correction to the $\mathrm{RH}$ conditions predicts variations in the flow velocity $u_{d}$ and in the speed of sound $a_{d}$, the detonation propagation velocity must provide $\delta\left\langle\mathcal{M}_{d}\right\rangle=0=\bar{\epsilon}^{2} \delta \mathcal{M}+\left(\mathrm{d} \mathcal{M}_{d}\right) /\left(\mathrm{d} u_{u}\right) \delta u_{u}$, and then

$$
\left.\delta \mathcal{S}\right|_{\delta\left\langle\mathcal{M}_{d}\right\rangle=0}=\left.\frac{1}{\bar{\epsilon}^{2}} \frac{\delta u_{u}}{u_{u}}\right|_{\delta\left\langle\mathcal{M}_{d}\right\rangle=0}=-\frac{1}{\sqrt{\mathcal{R}_{d} \mathcal{P}_{d}}}\left(\frac{\mathrm{d} \mathcal{M}_{d}}{\mathrm{~d} \mathcal{M}_{u}}\right)^{-1}\left(\delta \mathcal{U}+\frac{1}{2} \delta \mathcal{R}-\frac{1}{2} \delta \mathcal{P}\right)
$$

The variation of the propagation speed is studied through the order-of-unity factor $\delta \mathcal{S}$ as a function of the overdrive $\mathcal{M}_{u} / \mathcal{M}_{\mathrm{cj}}-1$, reduced heat release $\tilde{Q}$, and specific heat ratio $\gamma$ in Figs. 10, 11, and 12, respectively. To properly isolate the effects, heat release is conveniently redefined with the thermal enthalpy upstream $\tilde{Q}=q_{u} /\left(c_{p_{u}} T_{u}\right)$, which relates to $Q=\tilde{Q}(\gamma+1) / 2$ in the former definition.

Figure 10 shows the variation of the detonation propagation velocity given by 4.16 as a function of $\mathcal{M}_{u} / \mathcal{M}_{\mathrm{cj}}-1$ for three-dimensional isotropic perturbations, $\gamma=1.2$, $\tilde{Q}=1$ (a) and $\tilde{Q}=10$ (b). The four distinguished cases include $|W| \gg|H|$ (black lines), $|H| \gg|W|$ (green lines), $W=H$ (orange lines) and $W=-H$ (blue lines). For strongly overdriven detonations, dominant density perturbations induce the highest amplification to the propagation velocity. For iso-density mixtures, $|H| \gg|W|$ the correction of the propagation speed becomes negative for sufficiently overdriven detonations, thereby resulting in a slower propagation speed. However, this effect can be effectively neglected in view of its amplitude. Increasing the value of $\tilde{Q}$ results in higher values of $\delta \mathcal{S}$ for weakly overdriven detonations, except for $|W| \gg|H|$. Weakly overdriven detonations are dominated by heat-release perturbations, where the case $|H| \gg|W|$ predicts the highest amplification.

The effect of heat release is better analysed in Fig. 11. where the function $\delta \mathcal{S}$ is computed as a function of $\tilde{Q}$ for weakly overdriven (a) and strongly overdriven (b) detonations. The panel on the left shows that weakly overdriven detonations in het- 

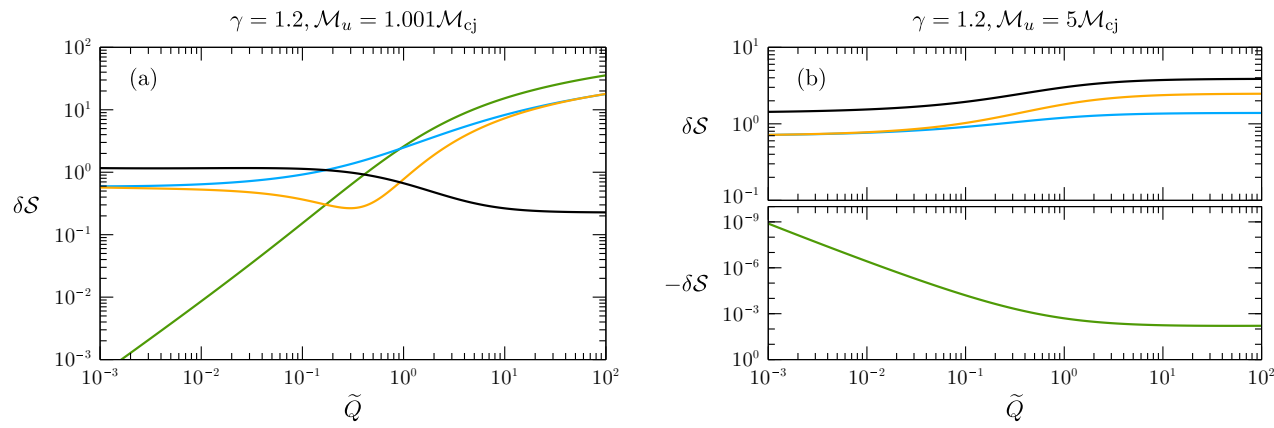

Figure 11: Three-dimensional second-order correction of the detonation propagation velocity $\delta \mathcal{S}$, according to 4.16 , as a function of the dimensionless heat release $\tilde{Q}$. Computations are shown for $\gamma=1.2$, weak-overdrives as $\mathcal{M}_{u}=1.001 \mathcal{M c j}$ (a) and strong-overdrives as $\mathcal{M}_{u}=5 \mathcal{M c j}(\mathrm{b}) .|W| \gg|H|(-),|H| \gg|W|(-), W=H(-)$ and $W=-H(-)$.
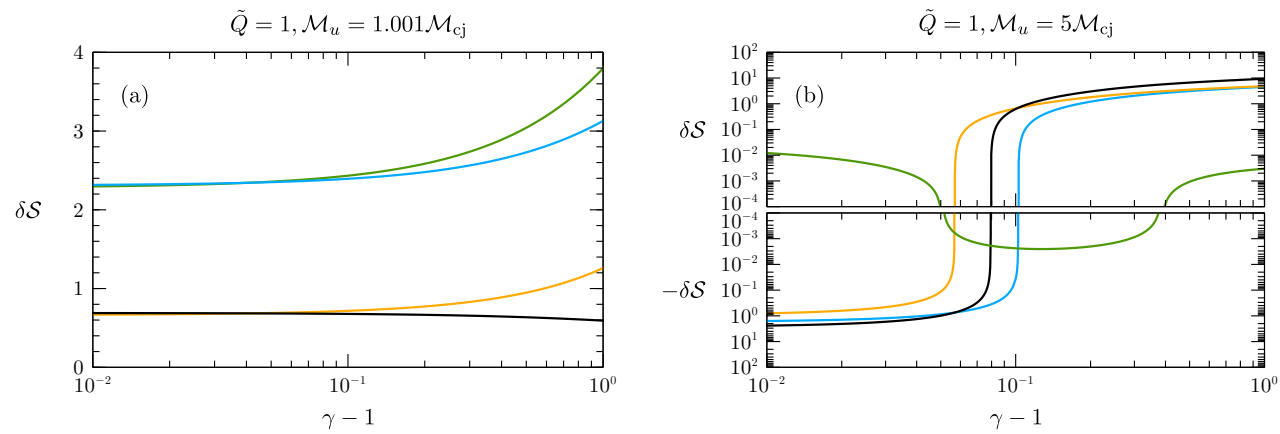

Figure 12: Three-dimensional second-order correction of the detonation propagation velocity $\delta \mathcal{S}$, according to 4.16), as a function of the adiabatic constant as $\gamma-1$. Computations are given for $Q=1$, weak-overdrives as $\mathcal{M}_{u}=1.001 \mathcal{M c j}$ (a) and strongoverdrives as $\mathcal{M}_{u}=5 \mathcal{M c j}(\mathrm{b})$. Computations are evaluated for $|W| \gg|H|(-)$, $|H| \gg|W|(-), W=H(-)$ and $W=-H(-)$.

erogeneous mixture tend to move faster for any value of $\tilde{Q}$. Regarding the type of mixture, higher heat release is associated with smaller amplification for $|W| \gg|H|$, while the opposite is found for $|H| \gg|W|$. Intermediate mixture properties $W=H$ and $W=-H$ present in-between values. For strongly overdriven detonations (b), the effect of heat release is to amplify the propagation speed when density perturbations are not negligible. However, a negative correction is found for iso-density mixtures dominated by heat-release perturbations $|H| \gg|W|$; however, the order of magnitude, which is significantly smaller, predicts a negligible correction to the propagation speed.

The effect of the specific heats ratio $\gamma$ is displayed in Fig. 12 , where the factor $\delta \mathcal{S}$ is computed as a function of $\gamma-1$ for weakly overdriven (a) and strongly overdriven (b) detonations. The former, in agreement with previous figures, shows an alwayspositive velocity correction. When density perturbations dominate the upstream flow, the reduction of $\gamma$ results in a higher propagation speed, but this trend reverses when heat release perturbations are included. Overdriven detonations, on the other hand, exhibit a less intuitive picture. For values of $\gamma$ between 1.1 and 1.4, the cases of utmost interest, results are qualitatively similar to those obtained previously for $\gamma=1.2$, where the case 
$|H| \gg|W|$ predicts minor negative corrections to the propagation speed, and the cases $|W| \gg|H|$ and $|W|=|H|$ predict positive order-of-unity values of $\delta \mathcal{S}$.

Based on the analysis performed in previous section, where the relative weight of the different type of perturbations in the burnt gas has been computed, it can be concluded that CJ-detonations moving in heterogeneous mixtures are governed by acoustic-type turbulent fluctuations in the burnt gas when net heat release is not negligible. Consequently, these factors are highly influenced by the heat-release variations derived from the upstream heterogeneities with the correlation with density perturbations playing a dominant role in the formation of turbulent structures downstream. These results are in qualitative agreement with previous numerical simulations (Mi et al. $2017 a b$ ); however, the numerical setup is not completely compatible with the assumptions made in this theoretical model. Spatial inhomogeneities are introduced by collecting the energy sources into discretized reactive layers and into reactive square-based prisms, both of which are spatially separated by inert regions $(\bar{\epsilon} \sim 1)$. They found that the average wave propagation velocity is greater, up to $10 \%$, than the CJ velocity of the equivalent homogeneous medium when the characteristic scale of the perturbations is greater than the inherent reaction zone length. The wave in CJ conditions speeds up when the specific heat ratio decreases. In agreement with linear theory, when the amplitude of the upstream heterogeneity increases the propagation speed also increases.

\section{Conclusions}

An analytical description of the linear interaction of a planar thin detonation front with a heterogeneous mixture of gases has been presented. The weak deviation of the fuel mass fraction with respect to the homogeneous case provides two sources of perturbations to the reactive Rankine-Hugoniot equations: the density relative changes associated to the different density of the upstream gas mixture $W$ and the relative heatrelease changes induced by the dependence of the heat release with the fuel mass fraction $H$. The combination of the two has a strong impact in the detonation response, and major differences in the perturbations are generated in the burnt gas associated to the corresponding relation between the two types of perturbations. This notion suggests that heat-release changes across the detonation cannot be neglected when modelling the interaction with non-perfect reactive mixtures, which places the equivalence ratio as a pivotal parameter in the analysis.

The results presented for all quantities of interest can be particularly useful in analysing the linear response of detonations to weak disturbances for conditions that render the basic planar solution stable provided that the characteristic sizes of the disturbances are much larger than the detonation thickness. For the interaction with a single-frequency perturbation field, the long-time amplitude of the perturbations can be written in terms of the characteristic oscillation frequency of the detonation front. Neutral conditions associated with the absence of pressure perturbations at the oscillating detonation or fully planar propagating detonations occur for distinguished conditions of reactive mixture associated to the density and heat release variations, $W$ and $H$, respectively.

Fourier superposition for two- and three-dimensional isotropic fields is used to obtain integral formulae for the amplification of the kinetic energy, enstrophy, and density fluctuations in the burnt gas. The effects of the propagation Mach number, overdrive, and mixture properties through $W$ and $H$ are identified, and the later plays a key role in the intensity of the turbulence generation. Regarding the type of upstream mixture, density perturbations dominate the turbulence generation for moderate-to-high overdrives, while heat release variations become relevant for weak overdrives. As a direct result, heat- 
release variations make the acoustic contribution the same order as the rotational and entropic perturbations. Therefore, linear interaction analysis suggests that the turbulent flow downstream cannot be assumed to be isobaric, and the dilatational part of the velocity gradient tensor cannot be neglected in CJ conditions. This conclusion deserves further analysis by means of high-accuracy numerical simulations.

Turbulence generation provides second-order corrections to the averaged RankineHugoniot jump conditions. While the pressure and density ratios are lower in the whole range of the parametric space, the post-detonation Mach number is amplified. As a direct implication, the propagation of the detonation front traveling in heterogeneous mixtures differs from that in homogeneous mixtures with the same averaged upstream properties. How intense this deviation is depends on the effective boundary condition in the burnt-gas flow. However, regardless of the closure expression (externally-imposed pressure, velocity or Mach number), the propagation speed is higher than that in the equivalent homogeneous case in the vast majority of cases, which agrees with previous results obtained numerically (Li et al. 2015: Mi et al. 2017 a b; ; Prakash \& Raman 2019). The second-order correction to the propagation speed is determined by the type of turbulence generated downstream. For overdriven detonations, the downstream acoustic contribution plays a subdominant role in favour of rotational-entropic disturbances, while the contrary applies for weakly overdriven detonations, for which the effect of upstream heat-release variations is of utmost importance.

\section{Acknowledgements}

Work produced with the support of a 2019 Leonardo Grant for Researchers and Cultural Creators, BBVA Foundation and project PID2019-108592RB-C41 (MICINN/ FEDER, UE). The authors are grateful to Dr. Martinez-Ruiz for the fruitful discussions.

\section{Appendix A. Normal mode analysis}

Computations of the turbulence kinetic energy, along with the second-order corrections to the Rankine-Hugoniot jump conditions and detonation propagation speed, call for the amplitudes of the flow variables in the burnt gas. These amplitudes, provided by the corresponding transfer functions across the detonation front for a given upstream perturbation, can be analytically derived by means of the Laplace Transform functions, as shown in Huete et al. (2013, 2017). However, as the interest is placed in the longtime dynamics, normal mode analysis offers a simpler form to obtain the asymptotic amplitudes of the burnt-gas perturbations. Thus, assuming that upstream perturbations are described by functions 2.13) and (2.14), the asymptotic far-field amplitudes for the pressure, density and velocity functions can be written as follows:

$$
\begin{aligned}
\hat{p}(\hat{x}, \hat{y}, \tau) & =\mathbb{P}_{a} e^{\mathrm{i}\left(\kappa_{a} \hat{x}-\omega_{a} \tau\right)} e^{\mathrm{i} \kappa_{y} \hat{y}}, \\
\hat{\rho}=\hat{\rho}_{a}(\hat{x}, \hat{y}, \tau)+\hat{\rho}_{e}(\hat{x}, \hat{y}) & =\left[\mathbb{D}_{a} e^{\mathrm{i}\left(\kappa_{a} \hat{x}-\omega_{a} \tau\right)}+\mathbb{D}_{e} e^{\mathrm{i} \kappa_{e} \hat{x}}\right] e^{\mathrm{i} \kappa_{y} \hat{y}}, \\
\hat{u}=\hat{u}_{a}(\hat{x}, \hat{y}, \tau)+\hat{u}_{r}(\hat{x}, \hat{y}) & =\left[\mathbb{U}_{a} e^{\mathrm{i}\left(\kappa_{a} \hat{x}-\omega_{a} \tau\right)}+\mathbb{U}_{r} e^{\mathrm{i} \kappa_{r} \hat{x}}\right] e^{\mathrm{i} \kappa_{y} \hat{y}}, \\
\hat{v}=\hat{v}_{a}(\hat{x}, \hat{y}, \tau)+\hat{v}_{r}(\hat{x}, \hat{y}) & =\left[\mathbb{V}_{a} e^{\mathrm{i}\left(\kappa_{a} \hat{x}-\omega_{a} \tau\right)}+\mathbb{V}_{r} e^{\mathrm{i} \kappa_{r} \hat{x}}\right] e^{\mathrm{i} \kappa_{y} \hat{y}},
\end{aligned}
$$

where the acoustic (subscript $a$ ), entropic (subscript $e$ ) and rotational (subscript $r$ ) contributions are easily recognized. The spatial and temporal acoustic frequencies are $\kappa_{a}$ and $\omega_{a}$, respectively, and the rotational/entropic spatial frequency is $\kappa_{r}=\kappa_{e}=\mathcal{R}_{d} \kappa_{x}$. 
While the value of the amplitudes must be solved with the aid of the linearized $\mathrm{RH}$ equations given in (2.17), the spatial and temporal frequencies are readily obtained by introducing the normal mode functions in the linear Euler equations 2.16 )

$$
\begin{aligned}
-\omega_{a} \mathbb{D}_{a}+\kappa_{a} \mathbb{U}_{a}+\kappa_{y} \mathbb{V}_{a}=0 \\
-\omega_{a} \mathbb{U}_{a}+\kappa_{a} \mathbb{P}_{a}=0, \\
-\omega_{a} \mathbb{V}_{a}+\kappa_{y} \mathbb{P}_{a}=0 \\
\mathbb{P}_{a}-\mathbb{D}_{a}=0
\end{aligned}
$$

thereby yielding the adiabatic dispersion relationship, $\omega_{a}^{2}=\kappa_{a}^{2}+\kappa_{y}^{2}$, of the flow perturbations in the burnt gas. As a result of the Doppler effect, the frequency seen by the detonation front is $\omega_{d}=\omega_{a}-\mathcal{M}_{d} \kappa_{a}$ when there is a single excitation frequency in the upstream heterogeneous flow (isolated and stable detonation assumption). This is used to give

$$
\begin{aligned}
& \kappa_{a}= \frac{\omega_{d} \mathcal{M}_{d}-\sqrt{\omega_{d}^{2}-\kappa_{y}^{2}\left(1-\mathcal{M}_{d}^{2}\right)}}{1-\mathcal{M}_{d}^{2}}, \\
& \omega_{a}=\frac{\omega_{d}-\mathcal{M}_{d} \sqrt{\omega_{d}^{2}-\kappa_{y}^{2}\left(1-\mathcal{M}_{d}^{2}\right)}}{1-\mathcal{M}_{d}^{2}},
\end{aligned}
$$

as the characteristic spatial and temporal frequencies of the downstream acoustic field.

When these parameters are real, the frequency $\omega_{a}$ is always positive, while the wavenumber $\kappa_{a}$ can be either positive or negative depending on whether the frequency of oscillation $\omega_{d}$ is smaller or larger than $\kappa_{y}$. Thus, from A 1 it is observed that $\kappa_{a}>0$ corresponds to right-traveling waves, while left-traveling waves prevail for $\kappa_{a}<0$. In a laboratory frame where the upstream flow remains static, the latter condition refers to the case where the sound waves escaping from the detonation front surmount the velocity of the burnt-gas fluid particles and propagate downstream. The other distinguished case occurs when the values of $\kappa_{a}$ and $\omega_{a}$ become imaginary, thereby yielding an exponential decay of the acoustic disturbances behind the front, a non-radiating condition that holds whenever $\omega_{d}<\kappa_{y}\left(1-\mathcal{M}_{d}^{2}\right)^{1 / 2}$. In the opposite case, the detonation is said to be in the radiation regime, a condition that is hard to meet when there is no external excitation, as shown in Huete \& Vera (2019).

Direct combination of the linearized $\mathrm{RH}$ equations with the inviscid-inert Euler equations in the burnt-gas state gives the dispersion relation

$$
\begin{array}{r}
{\left[\omega_{a}^{2}\left(\sigma_{b}+\mathcal{M}_{d}\right)-\kappa_{a} \omega_{a}\left(\mathcal{M}_{d}^{2}+2 \mathcal{M}_{d} \sigma_{b}+1\right)+\kappa_{a}^{2} \mathcal{M}_{d}\left(\mathcal{M}_{d} \sigma_{b}+1\right)-\kappa_{y}^{2}\left(1-\mathcal{M}_{d}^{2}\right) \sigma_{c}\right] \mathbb{P}_{a}} \\
=\alpha\left[\omega_{d}^{2}-\kappa_{y}^{2}\left(1-\mathcal{M}_{d}^{2}\right) \sigma_{d}\right]
\end{array}
$$

for the pressure fluctuations at the detonation front, where

$$
\sigma_{b}=\frac{1+\Gamma}{2 \mathcal{M}_{d}}, \sigma_{c}=\frac{\mathcal{M}_{d}^{2} \mathcal{R}_{d}}{1-\mathcal{M}_{d}^{2}} \frac{1-\Gamma}{2 \mathcal{M}_{d}}, \sigma_{d}=\frac{\mathcal{M}_{d}^{2} \mathcal{R}_{d}}{1-\mathcal{M}_{d}^{2}} \frac{\left(\mathcal{R}_{d}+\Delta-2\right) W-(1-\Delta) H}{\left(\mathcal{R}_{d}-\Delta\right) W+(1-\Delta) H},
$$

and the function

$$
\alpha=\frac{\mathcal{M}_{d}\left[\left(\mathcal{R}_{d}-\Delta\right) W+(1-\Delta) H\right]}{2 \sqrt{W^{2}+H^{2}}}
$$

accounts for the effective amplitude of the upstream excitation.

By employing normal mode analysis, the long-time dynamics of the pressure perturbation can be written as a piece-wise function of the high-frequency (sub-index $h$ ) and 
low-frequency (sub-indices $l_{1}$ and $l_{2}$ ) contributions

$$
\begin{array}{ll}
\hat{p}_{d}(\tau \gg 1)=\mathbb{P}_{h} e^{-\mathrm{i} \omega_{d} \tau+\mathrm{i} \kappa_{y} \hat{y}} & \text { for } \quad \omega_{d} \geqslant \kappa_{y}\left(1-\mathcal{M}_{d}^{2}\right)^{1 / 2}, \\
\hat{p}_{d}(\tau \gg 1)=\sqrt{\mathbb{P}_{l_{1}}^{2}+\mathbb{P}_{l_{2}}^{2}} e^{-\mathrm{i}\left(\omega_{d} \tau+\phi_{a}\right)+\mathrm{i} \kappa_{y} \hat{y}} & \text { for } \quad \omega_{d} \leqslant \kappa_{y}\left(1-\mathcal{M}_{d}^{2}\right)^{1 / 2},
\end{array}
$$

with the phase $\tan \phi_{a}=\mathbb{P}_{l_{2}} / \mathbb{P}_{l_{1}}$ and the corresponding amplitudes $\mathbb{P}_{h}, \mathbb{P}_{l_{1}}$, and $\mathbb{P}_{l_{2}}$ given by

$$
\begin{aligned}
& \mathbb{P}_{h}=\frac{\alpha\left(\zeta^{2}-\sigma_{d}\right)}{\zeta \sqrt{\zeta^{2}-1}+\sigma_{b} \zeta^{2}-\sigma_{c}}, \\
& \frac{\mathbb{P}_{l_{1}}}{\left(\sigma_{b} \zeta^{2}-\sigma_{c}\right)}=\frac{-\mathbb{P}_{l_{2}}}{\zeta \sqrt{1-\zeta^{2}}}=\frac{\alpha\left(\zeta^{2}-\sigma_{d}\right)}{\zeta^{2}\left(1-\zeta^{2}\right)+\left(\sigma_{b} \zeta^{2}-\sigma_{c}\right)^{2}}
\end{aligned}
$$

with use made of the normalized dimensionless frequency

$$
\zeta=\frac{\omega_{d}}{\kappa_{y} \sqrt{1-\mathcal{M}_{d}^{2}}}=\frac{\mathcal{R}_{d} \mathcal{M}_{d}}{\sqrt{1-\mathcal{M}_{d}^{2}}} \frac{1}{\tan \theta}=\frac{\mathcal{R}_{d} \mathcal{M}_{d}}{\sqrt{1-\mathcal{M}_{d}^{2}}} \frac{\kappa_{x}}{\kappa_{y}} .
$$

It is readily seen that the critical frequency $\omega_{d}=\kappa_{y}\left(1-\mathcal{M}_{d}^{2}\right)^{1 / 2}$ corresponds to $\zeta=1$ and to the critical angle

$$
\tan \theta_{c}=\frac{\mathcal{R}_{d} \mathcal{M}_{d}}{\sqrt{1-\mathcal{M}_{d}^{2}}} .
$$

Inspection of the above equations provides conditions where the detonation travels through the monochromatic heterogeneous mixture without generating any pressure perturbations behind it, $\hat{p}_{d}=0$. These conditions are obtained by analysing the righthand side of (A 5), which becomes identically zero when upstream heat-release and density changes are correlated in the following form:

$$
\left.\frac{H}{W}\right|^{\mathbb{P}_{a}=0}=-\frac{\left(\mathcal{R}_{d}-\Delta\right) \omega_{d}^{2}-\mathcal{M}_{d}^{2} \mathcal{R}_{d}\left(\mathcal{R}_{d}+\Delta-2\right) \kappa_{y}^{2}}{(1-\Delta)\left(\omega_{d}^{2}+\mathcal{M}_{d}^{2} \mathcal{R}_{d} \kappa_{y}^{2}\right)} .
$$

When this condition is met, the detonation oscillates in mechanical equilibrium and no pressure perturbations are generated, a situation often referred to as a neutral condition. It is seen that $H / W$ is not an exclusive property of the detonation, as it also depends on the excitation frequency $\omega_{d} / k_{y}$. This dependence is computed in Fig. 13 (a) as a function of the incident wave angle $\theta$. Very large values of $|H / W|$ are representative of iso-density gas mixtures, while values of $|H / W| \ll 1$ represent situations close to the peak in the heat release-fuel mass fraction diagram, which is usually placed in slightly rich mixtures. For slow-frequency oscillations, $\omega_{d} / \kappa_{y} \ll 1$ or $\theta \sim 90^{\circ}$, the detonation behaves neutrally if

$$
\left.\frac{H}{W}\right|_{\pi / 2-\theta \ll 1} ^{\mathbb{P}_{a}=0}=\frac{\mathcal{R}_{d}+\Delta-2}{1-\Delta},
$$

which represents a positive correlation between the changes in density and heat release. On the other hand, high-frequency perturbations $\omega_{d} \gg \kappa_{y}$ can render a neutral state only in negatively-correlated conditions, namely

$$
\left.\frac{H}{W}\right|_{\theta \ll 1} ^{\mathbb{P}_{a}=0}=-\frac{\mathcal{R}_{d}-\Delta}{1-\Delta} .
$$

Both limiting conditions tend to infinity in the strongly overdriven limit as $\Delta \sim 1-$ $O\left(\mathcal{M}_{d} / \mathcal{M}_{\mathrm{cj}}\right)$. 


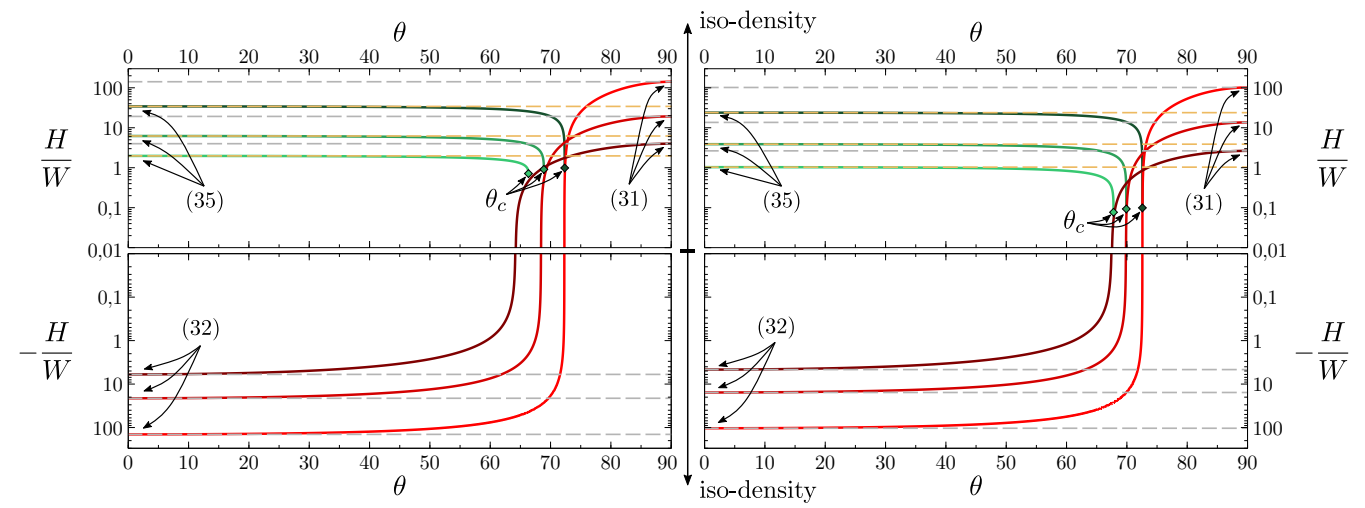

Figure 13: Iso-curves $\mathbb{P}_{a}=0$ (light-to-dark red as the incident Mach number increases) and $\mathbb{X}=0$ (light-to-dark green as the incident Mach number increases) as a function of the correlation factor $H / W$ and the incident angle $\theta=\tan ^{-1}\left(\kappa_{y} / \kappa_{x}\right)$ for $\gamma=1.2, \mathcal{M}_{u}=$ $(1.2,2,5) \times \mathcal{M}_{\mathrm{cj}}$ and $Q=1$ (left) and $Q=10$ (right).

Similarly, the amplitude of the perturbations of the detonation front is computed using (2.17a) to yield

$$
\begin{array}{ll}
\xi_{d}(\tau \gg 1)=\mathbb{X}_{h} e^{-\mathrm{i} \omega_{d} \tau+\mathrm{i} \kappa_{y} \hat{y}} & \text { for } \quad \omega_{d} \geqslant \kappa_{y}\left(1-\mathcal{M}_{d}^{2}\right)^{1 / 2}, \\
\xi_{d}(\tau \gg 1)=\sqrt{\mathbb{X}_{l_{1}}^{2}+\mathbb{X}_{l_{2}}^{2}} e^{-\mathrm{i}\left(\omega_{d} \tau+\phi_{\xi}\right)+\mathrm{i} \kappa_{y} \hat{y}} & \text { for } \quad \omega_{d} \leqslant \kappa_{y}\left(1-\mathcal{M}_{d}^{2}\right)^{1 / 2},
\end{array}
$$

where $\tan \phi_{\xi}=\mathbb{X}_{l_{2}} / \mathbb{X}_{l_{1}}$ and the functions $\mathbb{X}_{h}, \mathbb{X}_{l_{1}}$ and $\mathbb{X}_{l_{2}}$ can be explicitly written, with the aid of $(2.17 a)$, as a function of the pressure function

$$
\begin{aligned}
\mathbb{X}_{h} & =\frac{\mathcal{R}_{d} \mathcal{M}_{d}}{\zeta\left(\mathcal{R}_{d}-1\right) \sqrt{1-\mathcal{M}_{d}^{2}}}\left[\frac{1-\Gamma}{2 \mathcal{M}_{d}^{2}} \mathbb{P}_{h}-\frac{\left(\mathcal{R}_{d}+\Delta-2\right) W-(1-\Delta) H}{2 \sqrt{W^{2}+H^{2}}}\right], \\
\mathbb{X}_{l_{1}} & =\frac{\mathcal{R}_{d} \mathcal{M}_{d}}{\zeta\left(\mathcal{R}_{d}-1\right) \sqrt{1-\mathcal{M}_{d}^{2}}}\left[\frac{1-\Gamma}{2 \mathcal{M}_{d}^{2}} \mathbb{P}_{l_{1}}-\frac{\left(\mathcal{R}_{d}+\Delta-2\right) W-(1-\Delta) H}{2 \sqrt{W^{2}+H^{2}}}\right], \\
\mathbb{X}_{l_{2}} & =-\frac{\mathcal{R}_{d} \mathcal{M}_{d}}{\zeta\left(\mathcal{R}_{d}-1\right) \sqrt{1-\mathcal{M}_{d}}} \frac{1-\Gamma}{2 \mathcal{M}_{d}} \mathbb{P}_{l_{2}} .
\end{aligned}
$$

The neutral condition for the wrinkled detonation front, $\mathbb{X}=0$, can also be computed, and is shown in Fig. 13 in green colours. Asymptotic planar detonations occur in the highfrequency regime, for $\theta<\theta_{c r}$, with the correlation value that predicts $\mathbb{X}=0$ approaching the case for $\mathbb{P}_{a}=0$ when $\theta \sim \theta_{c r}$; however, they cannot occur simultaneously. In the high-frequency limit $\omega_{d} \gg \kappa_{y}$ the correlation factor reaches an asymptotic value given by

$$
\left.\frac{H}{W}\right|_{\theta \ll 1} ^{\mathbb{X}=0}=\frac{\left(\mathcal{R}_{d}-1\right)\left(\mathcal{M}_{d}+\Gamma\right)}{(1-\Delta)\left(1+\mathcal{M}_{d}\right)}-1,
$$

which, unlike the case for $\mathbb{P}=0$, is positive. Along the green curves, the detonation front is negligibly distorted, while dimensionless pressure perturbations are of the order of $\epsilon$.

As the detonation propagates through the heterogeneous mixture, various types of perturbations are generated downstream. As shown in (A 1), the terms can be decomposed into acoustic, rotational and entropic fluctuations Kovasznay (1953); Chu \& Kovásznay (1958), with the former taking the form of adiabatic traveling waves. The values of $\kappa_{a}$ and $\omega_{a}$ turn out to be imaginary for $\omega_{d} \leqslant \kappa_{y}\left(1-\mathcal{M}_{d}^{2}\right)^{1 / 2}$, which translates into an 
effectively null acoustic contribution in the far-field burnt gas. This is readily seen in a reference frame attached to the detonation front $\hat{x}_{s}=\mathcal{M}_{d} \tau-\hat{x}$, which provides the following pressure field

$$
\hat{p}\left(\hat{x}_{s}, \hat{y}, \tau\right)=\sqrt{\mathbb{P}_{l_{1}}^{2}+\mathbb{P}_{l_{2}}^{2}} e^{-\mathrm{i} \omega_{d}\left(\tau+\hat{x}_{s} \frac{\mathcal{M}_{d}}{1-\mathcal{M}_{d}^{2}}+\phi_{a}\right)+\mathrm{i} \kappa_{y} \hat{y}} e^{-\sigma(\theta) \hat{x}_{s}}
$$

for low-frequency oscillations, where

$$
\sigma(\theta)=\frac{\sqrt{\left(1-\mathcal{M}_{d}^{2}\right) \sin ^{2} \theta-\mathcal{R}_{d}^{2} \mathcal{M}_{d}^{2} \cos ^{2} \theta}}{1-\mathcal{M}_{d}^{2}}
$$

defines the spatial decay rate that varies from 0 (for $\theta=\theta_{c}$ ) to $1 / \sqrt{1-\mathcal{M}_{d}^{2}}$ (for $\theta=\pi / 2$ ). It is observed that the maximum decay rate increases as the overdrive decreases. For $\omega_{d} \geqslant \kappa_{y}\left(1-\mathcal{M}_{d}^{2}\right)^{1 / 2}$, the pressure field in the burnt gas is given by

$$
\hat{p}(\hat{x}, \hat{y}, \tau)=\mathbb{P}_{h} e^{-\mathrm{i}\left(\omega_{a} \tau-\kappa_{a} \hat{x}\right)+\mathrm{i} \kappa_{y} \hat{y}},
$$

where the amplitude corresponds to that at the detonation front, as in (A 8). The isentropic density variations induced by the acoustic radiation are $\mathbb{D}_{a}=\mathbb{P}_{h}$, as dictated by A $2 d$. Similarly, the associated velocity perturbations of the sonic wave emitted by the detonation can be computed with the aid of $(\mathrm{A} 2 b$ and $(\mathrm{A} 2 c)$, which gives $\mathbb{U}_{a}=\mathbb{P}_{h} \kappa_{a} / \omega_{a}$ and $\mathbb{V}_{a}=\mathbb{P}_{h} \kappa_{y} / \omega_{a}$ for the longitudinal and transverse contributions, respectively.

The acoustic velocity perturbations, which reach the far field only for $\zeta>1\left(\theta<\theta_{c}\right)$, are shown in Fig. 14 in the form of log-polar plots of $\left|\mathbb{U}_{a}\right|$ (left panels) and $\left|\mathbb{V}_{a} \tan \theta\right|$ (right panels) as functions of the incident wave angle $\theta$. The results are computed for $\gamma=1.2, \mathcal{M}_{u}=1.01 \mathcal{M}_{\mathrm{cj}}(\mathrm{a}-\mathrm{b}), \mathcal{M}_{u}=1.2 \mathcal{M}_{\mathrm{cj}}(\mathrm{c}-\mathrm{d})$ and $\mathcal{M}_{u}=2 \mathcal{M}_{\mathrm{cj}}$ (e-f) and show curves corresponding to $|W| \gg|H|$ (black lines), $|H| \gg|W|$ (green lines), $W=H$ (orange lines) and $W=-H$ (blue lines).

Computations show that the radiated acoustic energy is very sensitive to the ratio between $W$ and $H$, specially for weak overdrives. For example, when density variations are negligible, $|H| \gg|W|$, the acoustic perturbations are one order of magnitude smaller for finite overdrives, but they are non-negligible for $\mathcal{M}_{u}=1.01 \mathcal{M}_{\mathrm{cj}}$. It is also observed that positive correlations $W=H$ lead to more intense acoustic perturbations than negative correlations $W=-H$, but with the increase of overdrive both tend to the same value. In all cases, the maximum acoustic intensity is generated when $\theta \sim \theta_{c}$. It is also found that $\left|\mathbb{U}_{a}\right|$ and $\left|\mathbb{V}_{a} \tan \theta\right|$ become null when $\mathbb{P}=0$, a condition met when $\mathrm{A} 12$ is satisfied. The intermediate angle at which acoustic perturbations cancel out approaches $\theta_{c}$ when the overdrive increases, as observed in Fig. 13.

Along with the acoustic field, the oscillating detonation front is a source of entropy and vorticity disturbances. The former

$$
\begin{array}{ll}
\hat{\rho}_{e}(\hat{x} \gg 1, \hat{y})=\mathbb{D}_{e, h} e^{\mathrm{i} \kappa_{e} \hat{x}+\mathrm{i} \kappa_{y} \hat{y}} & \text { for } \quad \omega_{d} \geqslant \kappa_{y}\left(1-\mathcal{M}_{d}^{2}\right)^{1 / 2}, \\
\hat{\rho}_{e}(\hat{x} \gg 1, \hat{y})=\sqrt{\mathbb{D}_{e, l_{1}}^{2}+\mathbb{D}_{e, l_{2}}^{2} e^{\mathrm{i}\left(\kappa_{e} \hat{x}+\phi_{e}\right)+\mathrm{i} \kappa_{y} \hat{y}}} \quad \text { for } \quad \omega_{d} \leqslant \kappa_{y}\left(1-\mathcal{M}_{d}^{2}\right)^{1 / 2}
\end{array}
$$

where $\tan \phi_{e}=\mathbb{D}_{e, l_{2}} / \mathbb{D}_{e, l_{1}}$ and $\kappa_{e}=\mathcal{R}_{d} \kappa_{x}$, is easily evaluated by subtracting the acoustic 

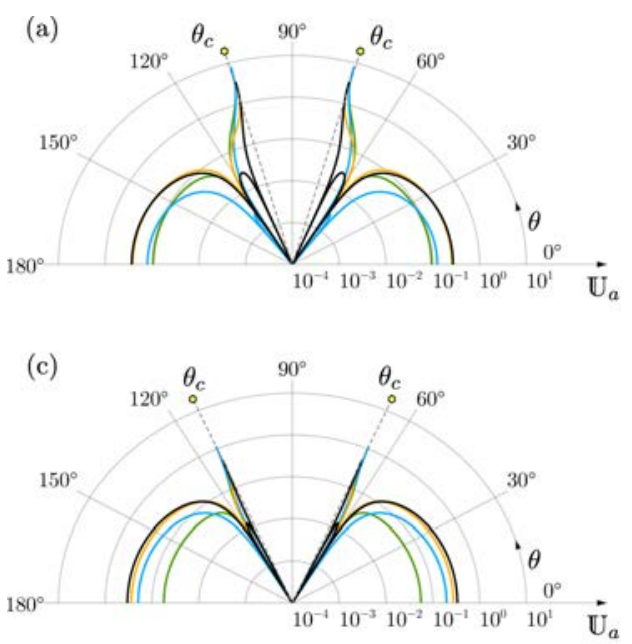

(e) $\quad \begin{array}{rrr}\theta_{c} & 90^{\circ} \quad \theta_{c} \\ & 120^{\circ} & \end{array}$

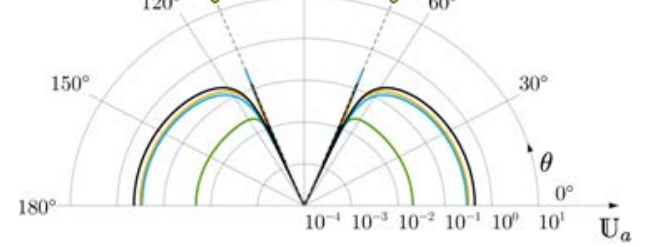

- $|W| \gg|H|$

घ $|H| \gg|W|$
A. Cuadra et al.
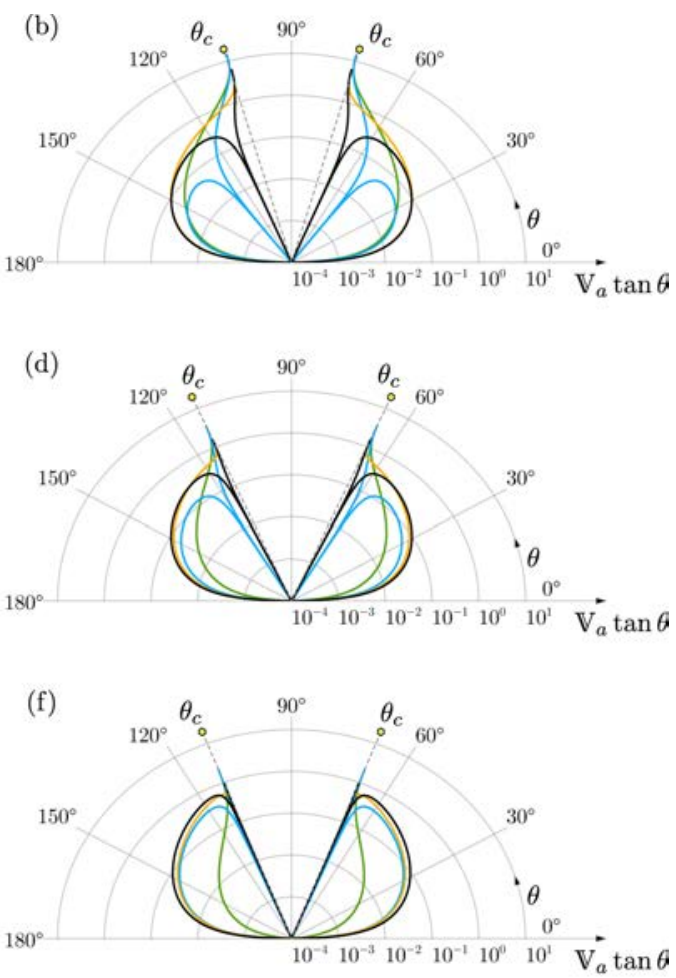

ㅁ $W=H$

ㅁ $W=-H$

Figure 14: Log-polar plot for $\left|\mathbb{U}_{a}\right|$ (a, c and e) and $\left|\mathbb{V}_{a} \tan \theta\right|(b, d$ and $\mathrm{f}$ ) as functions of the incident wave angle $\theta=\tan ^{-1}\left(\kappa_{y} / \kappa_{x}\right)$ for $\gamma=1.2, \mathcal{M}_{u}=1.01 \mathcal{M}_{\mathrm{cj}}(\mathrm{a}-\mathrm{b}), \mathcal{M}_{u}=1.2 \mathcal{M}_{\mathrm{cj}}$ (c-d), $\mathcal{M}_{u}=2 \mathcal{M}_{\mathrm{cj}}(\mathrm{e}-\mathrm{f}), Q=1$, and $|W| \gg|H|(-),|H| \gg|W|(-), W=H(-)$

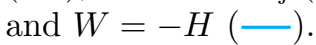

contribution in the $\mathrm{RH}$ equation $2.17 c$. The corresponding amplitudes are

$$
\begin{aligned}
\mathbb{D}_{e, h} & =\frac{\Gamma-\mathcal{M}_{d}^{2}}{\mathcal{M}_{d}^{2}} \mathbb{P}_{h}+\frac{\Delta W-(1-\Delta) H}{\sqrt{W^{2}+H^{2}}}, \\
\mathbb{D}_{e, l_{1}} & =\frac{\Gamma-\mathcal{M}_{d}^{2}}{\mathcal{M}_{d}^{2}} \mathbb{P}_{l_{1}}+\frac{\Delta W-(1-\Delta) H}{\sqrt{W^{2}+H^{2}}}, \\
\mathbb{D}_{e, l_{2}} & =\frac{\Gamma-\mathcal{M}_{d}^{2}}{\mathcal{M}_{d}^{2}} \mathbb{P}_{l_{2}},
\end{aligned}
$$

for the high frequency (short wavelength) and low frequency (long wavelength) regimes, respectively.

The dimensionless vorticity function $\Omega(\hat{x}, \hat{y}) \kappa_{y}=\partial \hat{v} / \partial \hat{x}-\partial \hat{u} / \partial \hat{y}$ is computed by calculating the vorticity generated by the first-order perturbations on the corrugated front, namely,

$$
\frac{\partial \hat{v}}{\partial \hat{x}}-\frac{\partial \hat{u}}{\partial \hat{y}}=-\frac{(1-\Gamma)\left(\mathcal{R}_{d}-1\right)}{2 \mathcal{M}_{d}} \frac{\partial \hat{p}_{d}}{\partial \hat{y}}+\frac{\mathcal{M}_{d}\left(\mathcal{R}_{d}-1\right)}{2} \frac{\left(\mathcal{R}_{d}+\Delta\right) W-(1-\Delta) H}{\sqrt{W^{2}+H^{2}}} \frac{\partial \delta Y_{u}}{\partial \hat{y}},
$$

where the functions $\hat{p}_{d}$ and $\delta Y_{u}$ are evaluated at the detonation front $\tau=\hat{x} / \mathcal{M}_{d}$. The 

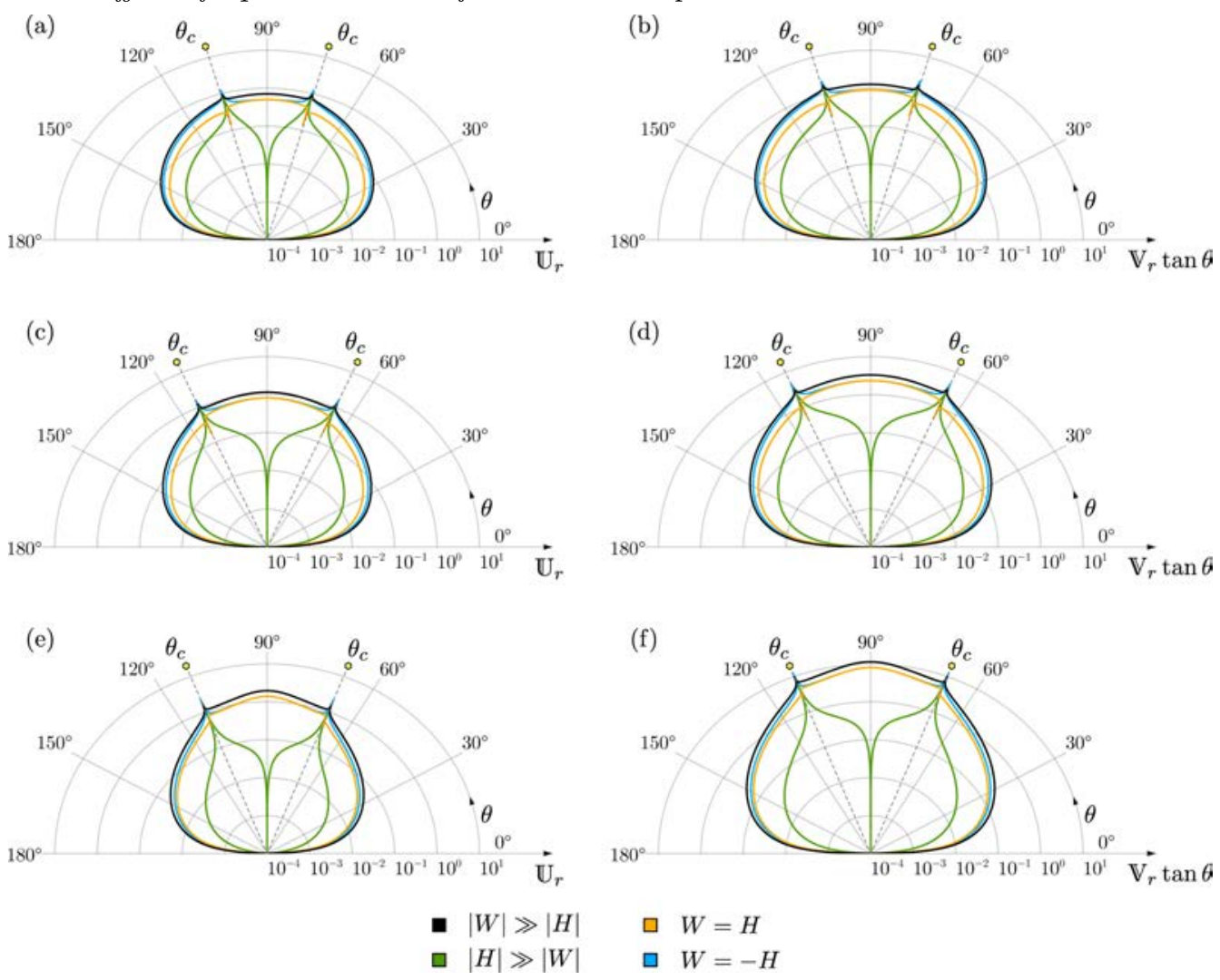

Figure 15: Log-polar plot for $\left|\mathbb{U}_{r}\right|$ (a,c and e) and $\left|\mathbb{V}_{r} \tan \theta\right|(b, d$, and $\mathrm{f}$ ) as functions of the incident wave angle $\theta=\tan ^{-1}\left(\kappa_{y} / \kappa_{x}\right)$ for $\gamma=1.2, \mathcal{M}_{u}=1.01 \mathcal{M}_{\mathrm{cj}}(\mathrm{a}-\mathrm{b}), \mathcal{M}_{u}=1.2 \mathcal{M}_{\mathrm{cj}}$ (c-d), $\mathcal{M}_{u}=2 \mathcal{M}_{\mathrm{cj}}(\mathrm{e}-\mathrm{f}), Q=1$, and $|W| \gg|H|(-),|H| \gg|W|(-), W=H(-)$ and $W=-H(-)$.

vorticity generated remains constant in the absence of diffusive effects, thereby yielding

$$
\begin{array}{ll}
\Omega(\hat{x} \gg 1, \hat{y})=\mathbb{O}_{h} e^{\mathrm{i} \kappa_{r} \hat{x}+\mathrm{i} \kappa_{y} \hat{y}} & \text { for } \quad \omega_{d} \geqslant \kappa_{y}\left(1-\mathcal{M}_{d}^{2}\right)^{1 / 2}, \\
\Omega(\hat{x} \gg 1, \hat{y})=\sqrt{\mathbb{O}_{l_{1}}^{2}+\mathbb{O}_{l_{2}}^{2}} e^{\mathrm{i}\left(\kappa_{r} \hat{x}+\phi_{r}\right)+\mathrm{i} \kappa_{y} \hat{y}} & \text { for } \quad \omega_{d} \leqslant \kappa_{y}\left(1-\mathcal{M}_{d}^{2}\right)^{1 / 2},
\end{array}
$$

for the far-field vorticity, where $\tan \phi_{r}=\mathbb{O}_{l_{2}} / \mathbb{O}_{l_{1}}$ and $\kappa_{r}=\kappa_{e}=\mathcal{R}_{d} \kappa_{x}$. The corresponding amplitudes of the associated short and long wavelengths are

$$
\begin{aligned}
\mathrm{O}_{h} & =\frac{(1-\Gamma)\left(\mathcal{R}_{d}-1\right)}{2 \mathcal{M}_{d}} \mathbb{P}_{h}-\frac{\mathcal{M}_{d}\left(\mathcal{R}_{d}-1\right)}{2} \frac{\left(\mathcal{R}_{d}+\Delta\right) W-(1-\Delta) H}{\sqrt{W^{2}+H^{2}}} \\
\mathbb{O}_{l_{1}} & =\frac{(1-\Gamma)\left(\mathcal{R}_{d}-1\right)}{2 \mathcal{M}_{d}} \mathbb{P}_{l_{1}}-\frac{\mathcal{M}_{d}\left(\mathcal{R}_{d}-1\right)}{2} \frac{\left(\mathcal{R}_{d}+\Delta\right) W-(1-\Delta) H}{\sqrt{W^{2}+H^{2}}} \\
\mathbb{O}_{l_{2}} & =\frac{(1-\Gamma)\left(\mathcal{R}_{d}-1\right)}{2 \mathcal{M}_{d}} \mathbb{P}_{l_{2}}
\end{aligned}
$$

The related steady-rotational velocity perturbations, which are isobaric in the linear 
limit, are governed by

$$
\frac{\partial^{2} \hat{u}_{r}}{\partial \hat{x}^{2}}+\frac{\partial^{2} \hat{u}_{r}}{\partial \hat{y}^{2}}=-\frac{\partial \Omega}{\partial \hat{y}} \quad \text { and } \quad \frac{\partial^{2} \hat{v}_{r}}{\partial \hat{x}^{2}}+\frac{\partial^{2} \hat{v}_{r}}{\partial \hat{y}^{2}}=-\frac{\partial \Omega}{\partial \hat{x}},
$$

which provide the following far-field velocity functions for the longitudinal and transverse contributions, respectively

$$
\begin{array}{ll}
\hat{u}_{r}(\hat{x} \gg 1, \hat{y})=\mathbb{U}_{r, h} e^{\mathrm{i} \kappa_{r} \hat{x}+\mathrm{i} \kappa_{y} \hat{y}} & \text { for } \quad \omega_{d} \geqslant \kappa_{y}\left(1-\mathcal{M}_{d}^{2}\right)^{1 / 2}, \\
\hat{u}_{r}(\hat{x} \gg 1, \hat{y})=\sqrt{\mathbb{U}_{r, l_{1}}^{2}+\mathbb{U}_{r, l_{2}}^{2} e^{\mathrm{i}\left(\kappa_{r} \hat{x}+\phi_{r}\right)+\mathrm{i} \kappa_{y} \hat{y}}} & \text { for } \quad \omega_{d} \leqslant \kappa_{y}\left(1-\mathcal{M}_{d}^{2}\right)^{1 / 2},
\end{array}
$$

and

$$
\begin{array}{ll}
\hat{v}_{r}(\hat{x} \gg 1, \hat{y})=\mathbb{V}_{r, h} e^{\mathrm{i} \kappa_{r} \hat{x}+\mathrm{i} \kappa_{y} \hat{y}} & \text { for } \quad \omega_{d} \geqslant \kappa_{y}\left(1-\mathcal{M}_{d}^{2}\right)^{1 / 2}, \\
\hat{v}_{r}(\hat{x} \gg 1, \hat{y})=\sqrt{\mathbb{V}_{r, l_{1}}^{2}+\mathbb{V}_{r, l_{2}}^{2} e^{\mathrm{i}\left(\kappa_{r} \hat{x}+\phi_{r}\right)+\mathrm{i} \kappa_{y} \hat{y}}} & \text { for } \quad \omega_{d} \leqslant \kappa_{y}\left(1-\mathcal{M}_{d}^{2}\right)^{1 / 2},
\end{array}
$$

with the corresponding amplitudes proportional to the vorticity amplitude $\mathbb{O}_{j}$ through

$$
\mathbb{U}_{r, j}=\frac{\mathrm{O}_{j}}{1+\left(\kappa_{r} / \kappa_{y}\right)^{2}}=\frac{\mathrm{O}_{j} \mathcal{M}_{d}^{2}}{\mathcal{M}_{d}^{2}+\zeta^{2}\left(1-\mathcal{M}_{d}^{2}\right)}=\mathbb{V}_{r, j} \frac{\kappa_{y}}{\kappa_{r}}=\mathbb{V}_{r, j} \frac{\mathcal{M}_{d}}{\zeta \sqrt{1-\mathcal{M}_{d}^{2}}}
$$

The subscript $j$ may refer to the sub-indices $h$ or $l_{1}$ and $l_{2}$ for high frequency and lowfrequency contributions, respectively, as noted in previous equations.

The computed amplitudes of the rotational velocity perturbations are presented in Fig. 15 as a function of the polar coordinate $\theta$, with the left and right plots corresponding to $\left|\mathbb{U}_{r}\right|$ and $\left|\mathbb{V}_{r} \tan \theta\right|$, respectively. As in Fig. 14. the results are obtained for $\gamma=$ $1.2, \mathcal{M}_{u}=1.01 \mathcal{M}_{\mathrm{cj}}(\mathrm{a}-\mathrm{b}), \mathcal{M}_{u}=1.2 \mathcal{M}_{\mathrm{cj}}(\mathrm{c}-\mathrm{d})$ and $\mathcal{M}_{u}=2 \mathcal{M}_{\mathrm{cj}}$ (e-f) and show curves corresponding to $|W| \gg|H|$ (black lines), $|H| \gg|W|$ (green lines), $W=H$ (orange lines) and $W=-H$ (blue lines). It can be inferred that considering $|W|>0$ amplifies the rotational perturbations in most of the domain for all degrees of overdrive, a finding that contrasts with the acoustic contribution. It is also observed that increasing the detonation intensity (by increasing the overdrive, see lower panel) amplifies the transverse part but barely changes the streamwise contribution. As expected, the maximum rotational intensity is produced close to the critical angle $\theta_{c}$ where all amplitudes peak. Aside from the trivial conditions determined by $\theta=0^{\circ}$ or $180^{\circ}$, null rotational contribution, i.e., $\left|\mathbb{U}_{r}\right|=0$ and $\left|\mathbb{V}_{r} \tan \theta\right|=0$, are found to occur for transverse stratified gases, i.e. $\theta=90^{\circ}$, with similar density and outside of the heat-release peak.

The above results provide the dependence of the post-detonation perturbation amplitudes $\mathbb{P}(\theta), \mathbb{D}(\theta), \mathbb{O}(\theta), \mathbb{U}(\theta)$ and $\mathbb{V}(\theta)$ for the pressure, density, vorticity and velocity variables, respectively, as a function of the incident angle $\theta=\tan ^{-1}\left(\kappa_{y} / \kappa_{x}\right)$. They give the amplitudes of the perturbations in the asymptotic far field when the oscillating detonation reaches the constant-amplitude regime governed by the external single-frequency excitation. This case is representative of scenarios where the upstream spectrum is dominated by a single characteristic frequency $k$, something that is not expected to occur in heterogeneous gaseous mixtures, but rather in multi-phase environments involving water sprays (Watanabe et al. 2019, 2020) or fuel sprays (Williams 1961). The model presented here cannot be directly applied to these conditions since in them density gradients cannot be linearized, and non-linear terms cannot be neglected. In general, specially in cases where the heterogeneities arise from the mixing of gaseous reactants as is considered here, the perturbations in the resulting mixture show a wide range of length scales. Then, the use of probability distribution functions is required to compute the flow perturbation variables downstream, as noted in Section 3 . 


\section{REFERENCES}

Andreopoulos, Yiannis, Agui, Juan H \& Briassulis, George 2000 Shock wave - turbulence interactions. Annual Review of Fluid Mechanics 32 (1), 309-345.

Batchelor, George Keith 1953 The theory of homogeneous turbulence. Cambridge university press.

Blackstock, DAvid T 1962 Propagation of plane sound waves of finite amplitude in nondissipative fluids. The Journal of the Acoustical Society of America 34 (1), 9-30.

Browne, S, Ziegler, J \& Shepherd, JE 2008 Shock and detonation toolbox. GALCITExplosion Dynamics Laboratory, Pasadena, CA .

Chu, BoA-Teh \& Kovásznay, Leslie SG 1958 Non-linear interactions in a viscous heatconducting compressible gas. Journal of Fluid Mechanics 3 (5), 494-514.

Clavin, Paul \& Williams, Forman A 2012 Analytical studies of the dynamics of gaseous detonations. Philosophical Transactions of the Royal Society A: Mathematical, Physical and Engineering Sciences 370 (1960), 597-624.

Ettner, F., Vollmer, K. G. \& Sattelmayer, T. 2013 Mach reflection in detonations propagating through a gas with a concentration gradient. Shock Waves 23 (3), 201-206.

Farag, G, Boivin, Pierre \& Sagaut, P 2019 Interaction of two-dimensional spots with a heat releasing/absorbing shock wave: linear interaction approximation results. Journal of Fluid Mechanics 871, 865-895.

Frolov, SM, Aksenov, VS, Ivanov, VS, Shamshin, IO \& Zangiev, AE 2019 Air-breathing pulsed detonation thrust module: Numerical simulations and firing tests. Aerospace Science and Technology 89, 275-287.

Goodwin, David G., Speth, Raymond L., Moffat, Harry K. \& Weber, Bryan W. 2018 Cantera: An object-oriented software toolkit for chemical kinetics, thermodynamics, and transport processes. https://www. cantera.org, version 2.4.0.

Gordon, SANFORd \& McBride, Bonnie J 1994 Computer program for calculation of complex chemical equilibrium compositions and applications. part 1: Analysis. NASA Reference Publication 1311 .

GRIfFOnd, J 2005 Linear interaction analysis applied to a mixture of two perfect gases. Physics of Fluids 17 (8), 086101.

Han, Wenhu, Wang, Cheng \& Law, Chung K 2019 Pulsation in one-dimensional h2-o2 detonation with detailed reaction mechanism. Combustion and Flame 200, 242-261.

Hazak, Giora, Velikovich, Alexander L, Gardner, John H \& Dahlburg, Jill P 1998 Shock propagation in a low-density foam filled with fluid. Physics of Plasmas 5 (12), 4357-4365.

Holzer, Mark \& Siggia, Eric D 1994 Turbulent mixing of a passive scalar. Physics of Fluids 6 (5), 1820-1837.

Huete, CÉsar, Jin, Tai, Martínez-Ruiz, Daniel \& Luo, Kun 2017 Interaction of a planar reacting shock wave with an isotropic turbulent vorticity field. Physical Review E 96 (5), 053104 .

Huete, César, Sánchez, Antonio L \& Williams, Forman A 2013 Theory of interactions of thin strong detonations with turbulent gases. Physics of Fluids 25 (7), 076105.

Huete, César, SÁnchez, Antonio L \& Williams, Forman A 2014 Linear theory for the interaction of small-scale turbulence with overdriven detonations. Physics of Fluids 26 (11), 116101.

Huete, César \& Vera, Marcos 2019 D'yakov-kontorovich instability in planar reactive shocks. Journal of Fluid Mechanics 879, 54-84.

Huff, Riley, Schauer, Frederick, Boller, Scott A, Polanka, Marc D, Fotia, MATThEW \& Hoke, John 2019 Exit condition measurements of a radial rotating detonation engine bleed air turbine. In AIAA Scitech 2019 Forum, p. 1011.

Hussein, SARAH Moussa 2018 Direct numerical simulation of homogeneous isotropic turbulence-a methodology and applications. PhD thesis, The University of Texas at Arlington.

JaCkson, TL, Hussaini, MY \& Ribner, HS 1993 Interaction of turbulence with a detonation wave. Physics of Fluids A: Fluid Dynamics 5 (3), 745-749.

Jackson, Thomas Luther, Kapila, Ashwani K \& Hussaini, M Yousuff 1990 Convection of 
a pattern of vorticity through a reacting shock wave. Physics of Fluids A: Fluid Dynamics $\mathbf{2}(7), 1260-1268$.

Jin, Tai, Luo, Kun, Dai, Qi \& FAn, Jianren 2016 Simulations of cellular detonation interaction with turbulent flows. AIAA Journal 54 (2), 419-433.

Kabanov, Dmitry I \& Kasimov, Aslan R 2018 Linear stability analysis of detonations via numerical computation and dynamic mode decomposition. Physics of Fluids 30 (3), 036103.

Kailasanath, K 2000 Review of propulsion applications of detonation waves. AIAA journal 38 (9), 1698-1708.

Kailasanath, K 2003 Recent developments in the research on pulse detonation engines. AIAA journal 41 (2), 145-159.

Kailasanath, K 2006 Liquid-fueled detonations in tubes. Journal of Propulsion and Power 22 (6), 1261-1268.

Kasimov, Aslan R \& Stewart, D Scott 2004 On the dynamics of self-sustained onedimensional detonations: A numerical study in the shock-attached frame. Physics of Fluids 16 (10), 3566-3578.

Kessler, DA, GAmezo, VN \& OrAn, ES 2012 Gas-phase detonation propagation in mixture composition gradients. Philosophical Transactions of the Royal Society A: Mathematical, Physical and Engineering Sciences 370 (1960), 567-596.

Kovasznay, Leslie SG 1953 Turbulence in supersonic flow. Journal of the Aeronautical Sciences 20 (10), 657-674.

Larsson, Johan \& Lele, SANJiva K 2009 Direct numerical simulation of canonical shock/turbulence interaction. Physics of fluids 21 (12), 126101.

Lee, Sangsan, Lele, Sanjiva K \& Moin, Parviz 1993 Direct numerical simulation of isotropic turbulence interacting with a weak shock wave. Journal of Fluid Mechanics 251, 533-562.

Lele, Sanjiva K 1992 Shock-jump relations in a turbulent flow. Physics of Fluids A: Fluid Dynamics 4 (12), 2900-2905.

Li, Jianling, Mi, Xiaocheng \& Higgins, Andrew J 2015 Effect of spatial heterogeneity on near-limit propagation of a pressure-dependent detonation. Proceedings of the Combustion Institute 35 (2), 2025-2032.

Livescu, DANiel 2020 Turbulence with large thermal and compositional density variations. Annual Review of Fluid Mechanics 52.

Massa, Luca, Chauhan, M \& Lu, FK 2011 Detonation-turbulence interaction. Combustion and flame 158 (9), 1788-1806.

Massa, LuCA \& Lu, Frank K 2011 The role of the induction zone on the detonation-turbulence linear interaction. Combustion Theory and Modelling 15 (3), 347-371.

Meadows, Joseph W \& Subramanian, Sathyanarayanan 2019 Novel approach for modeling non-premixed rotating detonation engine using a 2-d cfd analysis. In AIAA Propulsion and Energy 2019 Forum, p. 4130.

Meng, Qingyang, Zhao, Ningbo, Zheng, Hongtao, Yang, Jialong \& Qi, Lei 2018 Numerical investigation of the effect of inlet mass flow rates on h2/air non-premixed rotating detonation wave. International Journal of Hydrogen Energy 43 (29), 1361813631.

Mi, XiaoCheng, Higgins, Andrew J, Ng, Hoi Dick, Kiyanda, Charles B \& Nikiforakis, Nikolaos $2017 a$ Propagation of gaseous detonation waves in a spatially inhomogeneous reactive medium. Physical Review Fluids 2 (5), 053201.

Mi, XiaoCheng, Timofeev, Evgeny V \& Higgins, Andrew J $2017 b$ Effect of spatial discretization of energy on detonation wave propagation. Journal of Fluid Mechanics 817, 306-338.

Miller, RichaRd S 2000 Long time mass fraction statistics in stationary compressible isotropic turbulence at supercritical pressure. Physics of Fluids 12 (8), 2020-2032.

Ostrovskit, LA 1968 Second-order terms in a traveling sound wave. Soviet Physics-Acousctics 14, 61-66.

Prakash, Supraj, Fiévet, Romain \& Raman, Venkatramanan 2019a The effect of fuel stratification on the detonation wave structure. In AIAA Scitech 2019 Forum, p. 1511.

Prakash, Supraj, Fiévet, Romain, Raman, Venkat, Burr, Jason \& Yu, Kenneth H 
$2019 b$ Analysis of the detonation wave structure in a linearized rotating detonation engine. AIAA Journal pp. 1-15.

Prakash, Supraj \& Raman, S 2019 Detonation propagation through inhomogeneous fuelair mixtures. In International Colloquium on the Dynamics of Explosions and Reactive Systems, p. 81.

Pratt, David T, Humphrey, Joseph W \& Glenn, Dennis E 1991 Morphology of standing oblique detonation waves. Journal of Propulsion and Power 7 (5), 837-845.

Quadros, Russell, Sinha, Krishnendu \& Larsson, Johan 2016 Turbulent energy flux generated by shock/homogeneous-turbulence interaction. Journal of Fluid Mechanics 796, $113-157$.

Radulescu, Matei I, Sharpe, Gary J, Law, Chung K \& Lee, John HS 2007 The hydrodynamic structure of unstable cellular detonations. Journal of Fluid Mechanics 580, $31-81$.

Ribner, Herbert S 1954 a Convection of a pattern of vorticity through a shock wave. NACA TN-3255. Also NACA Rep. 1233. .

Ribner, HERBERT S 1954b Shock-turbulence interaction and the generation of noise, , vol. 1233. National Advisory Committee for Aeronautics.

Ribner, Herbert S 1987 Spectra of noise and amplified turbulence emanating from shockturbulence interaction. AIAA journal 25 (3), 436-442.

Sagaut, Pierre \& Cambon, Claude 2008 Homogeneous turbulence dynamics, , vol. 10. Springer.

Sethuraman, Yogesh \& Sinha, Krishnendu 2020 Modeling of thermodynamic fluctuations in canonical shock-turbulence interaction. AIAA Journal pp. 1-14.

Sethuraman, Yogesh Prasadd M, Sinha, Krishnendu \& Larsson, Johan 2018 Thermodynamic fluctuations in canonical shock-turbulence interaction: effect of shock strength. Theoretical and Computational Fluid Dynamics 32 (5), 629-654.

Shepherd, JE 2009 Detonation in gases. Proceedings of the Combustion Institute 32 (1), 83-98.

Short, MARK \& QUIRK, JAMES J 1997 On the nonlinear stability and detonability limit of a detonation wave for a model three-step chain-branching reaction. Journal of Fluid Mechanics 339, 89-119.

SinHA, KRISHNENDU 2012 Evolution of enstrophy in shock/homogeneous turbulence interaction. Journal of fluid mechanics 707, 74-110.

Tian, Yifeng, Jaberi, Farhad A \& Livescu, Daniel 2020 Modeling of shock propagation in non-uniform density media. In AIAA Scitech 2020 Forum, p. 0101.

URZAY, JAVIER 2018 Supersonic combustion in air-breathing propulsion systems for hypersonic flight. Annual Review of Fluid Mechanics 50, 593-627.

Velikovich, AL, Huete, C \& Wouchuk, JG 2012 Effect of shock-generated turbulence on the hugoniot jump conditions. Physical Review E 85 (1), 016301.

Veyssiere, B \& Khasainov, BA 1995 Structure and multiplicity of detonation regimes in heterogeneous hybrid mixtures. Shock Waves 4 (4), 171-186.

Watanabe, Hiroaki, Matsuo, Akiko, Chinnayya, Ashwin, Matsuoka, Ken, Kawasaki, AKIRA \& Kasahara, Jiro 2020 Numerical analysis of the mean structure of gaseous detonation with dilute water spray. Journal of Fluid Mechanics $\mathbf{8 8 7}$.

Watanabe, Hiroaki, Matsuo, Akiko, Matsuoka, Ken, Kawasaki, Akira \& Kasahara, JIRO 2019 Numerical investigation on propagation behavior of gaseous detonation in water spray. Proceedings of the Combustion Institute 37 (3), 3617-3626.

Westervelt, Peter J 1950 The mean pressure and velocity in a plane acoustic wave in a gas. The Journal of the Acoustical Society of America 22 (3), 319-327.

Williams, Forman A 1961 Structure of detonations in dilute sprays. The Physics of Fluids 4 (11), 1434-1443. 